University of Warwick institutional repository: http://go.warwick.ac.uk/wrap This paper is made available online in accordance with publisher policies. Please scroll down to view the document itself. Please refer to the repository record for this item and our policy information available from the repository home page for further information.

To see the final version of this paper please visit the publisher's website. Access to the published version may require a subscription.

Author(s): WEIXIAO SHEN

Article Title: On the measurable dynamics of real rational functions

Year of publication: 2003

Link to published

version: http://dx.doi.org/10.1017/S0143385702001311

Publisher statement: None 


\title{
On the measurable dynamics of real rational functions
}

\author{
WEIXIAO SHEN \\ Department of Mathematics, University of Warwick, Coventry CV4 7AL, UK \\ (e-mail:wxshen@maths.warwick.ac.uk)
}

(Received 13 December 2000 and accepted in revised form 26 June 2002)

\begin{abstract}
Let $f$ be a real rational function with all critical points on the extended real axis and of even order. Then:

(1) $f$ carries no invariant line field on the Julia set unless it is doubly covered by an integral torus endomorphism (a Lattés example); and

(2) $f \mid J(f)$ has only finitely many ergodic components.
\end{abstract}

\section{Introduction}

In this paper, we study the measurable dynamics of a real rational function $f$, which satisfies the following two conditions:

(C1) any critical point $c$ of $f$ is contained in $S^{1}=\hat{\mathbb{R}}=\mathbb{R} \cup\{\infty\}$;

(C2) any critical point $c$ of $f$ is of even order.

Let $\mathcal{F}$ denote the set of all real rational functions satisfying these two conditions. Our main result is the following theorem.

THEOREM 1. Any $f \in \mathcal{F}$ carries no invariant line field on the Julia set $J(f)$ unless it is a Lattés example.

THEOREM 2. Let $f \in \mathcal{F} . f \mid J(f)$ has only finitely many ergodic components.

It is conjectured that these theorems hold for all rational functions, see [16, 18, 22]. The question in the first main theorem is closely related to the well-known Fatou conjecture: in the space $\mathbf{R a t}_{d}$ of all rational maps (and in the space Poly $\mathbf{y}_{d}$ of all polynomials) of degree $d \geq 2$, hyperbolic ones form an open and dense subset. Our first main theorem implies the following corollary.

COROLLARY 3. Let $f$ be a real rational map of degree $d \geq 2$ for which all critical points are on the real axis. Suppose that $f$ is structurally stable in the space $\mathbf{R a t}_{d}$. Then $f$ is hyperbolic. 
Proof of Corollary 3. Since $f$ is structurally stable, the Teichmüller space of $f$ has dimension $2 d-2$. In particular, all the critical points are non-degenerate which implies that condition $(\mathrm{C} 2)$ holds. Thus $f \in \mathcal{F}$. It follows from Theorem 1 that $f$ carries no invariant line field on the Julia set. By Theorem 9.4 of [22], $f$ is hyperbolic.

Some progress in the direction of this work had been made by several authors, mostly on the case that $f$ is a quadratic polynomial. For a non-renormalizable polynomial, the absence of an invariant line field follows from the work of Yoccoz (see [9]), while McMullen [20] proved this for infinitely renormalizable quadratic polynomials which are 'robust', including all real ones. McMullen's method has been refined by Levin and van Strien [12] who generalized the result to all real unimodal polynomials. For polynomials with more critical points, the only result, to my knowledge, is in $[7,8]$, where the authors prove the absence of invariant line fields for a real polynomial with only real critical points and such that $f$ is infinitely renormalizable and of bounded type. The ergodic decomposition was proved by Lyubich [14] for a smooth one-dimensional dynamics with non-flat critical points and in the category of holomorphic dynamics, by Prado [25] for a real unimodal polynomial. In the proof of all these results, some kind of 'bound' plays a crucial role.

In this paper, Theorems 1 and 2 will be proved using the same method and the real bounds developed in [26] play a crucial role in our proof.

The main difficulty is to study the measurable dynamics on the part $J_{c}=\{z \in J(f)$ : $\omega(z)=\omega(c)\}$ for a recurrent critical point $c$ with a minimal $\omega$-limit set. It will be done in the same outline as in McMullen [20]. If a large real bound exists, then we shall transfer it to a 'complex bound' for a quasi-polynomial-like mapping, as in [12]. In the converse case, our argument will depend heavily on the essentially bounded geometry of the Cantor set $\omega(c)$. Comparing this work with that in [20], a new ingredient is the fact that the absence of a large real bound implies that $c$ is 'uniformly persistently recurrent' in some sense, which is also proved in [26].

Let us briefly outline the structure of this paper. Section 2 contains some technical preliminaries, including some distortion lemmas. In $\S 3$, we recall the definition and give some sufficient condition for the absence of invariant line fields. In $\S 4$, the notions of real box mappings and quasi-polynomial-like mappings are recalled. Section 5 is devoted to the study of the measurable dynamics on the subset of the Julia set consisting of points accumulating on a reluctantly recurrent critical point. It turns out that we will only need to consider the part $J_{c}$ for a persistently recurrent critical point. In $\S 6$, we study the dynamics of $f \mid J_{c}$ when a large bound exists for $c$. In $\S 7$, we shall study the converse case and then complete the proof of the main theorems.

\section{Preliminaries}

2.1. Distortion lemmas. We state some well-known distortion lemmas. Let $\Delta(r)=$ $\{z \in \mathbb{C}:|z|<r\}$ and let $\Delta=\Delta(1)$.

LEMMA 2.1. For any $\delta>0$ and $N \in \mathbb{N}$, there is a constant $\epsilon=\epsilon(N, \delta)$ such that the following holds. 
If $h: U \rightarrow V$ is a proper map of degree $N$ between two hyperbolic topological disks $U, V$, then for any $z_{0} \in U$, we have $h\left(\left\{z \in U: d_{U}\left(z, z_{0}\right)<\epsilon\right\}\right) \supset\{w \in V$ : $\left.d_{V}\left(h\left(z_{0}\right), w\right)<\delta\right\}$, where $d_{U}$ and $d_{V}$ are the hyperbolic metric of $U$ and $V$ respectively.

Moreover, $\epsilon \rightarrow 0$ as $\delta \rightarrow 0$ for a fixed $N$.

LEMMA 2.2. For any $0<r<1$ and $N \in \mathbb{N}$, there is a constant $C=C(r, N)>1$ with the following property.

If $U \subset \mathbb{C}$ is a topological disk and $h: U \rightarrow \Delta$ is a proper map of degree $\leq N$, then for any component $U_{0}$ of $h^{-1}(\Delta(r))$, we have $\operatorname{diam}\left(U_{0}\right)^{2} /$ area $\left(U_{0}\right) \leq C$, where the diameter and area are measured in the Euclidean metric of $U$.

LEMMA 2.3. For any $\delta>0$ and $N \in \mathbb{N}$, there is a constant $\epsilon=\epsilon(N, \delta)>0$ with the following property.

Let $U$ be a simply connected subset of $\hat{\mathbb{C}}$ and $h: U \rightarrow \Delta$ be a proper map of degree $\leq N$. Let $X \subset \Delta$ be a measurable set with $m(\Delta(1 / 2) \cap X) / m(\Delta(1 / 2)) \leq 1-\delta$, then for any component $U_{0}$ of $h^{-1}(\Delta(1 / 2))$, we have $m\left(U_{0} \cap h^{-1}(X)\right) / m\left(U_{0}\right) \leq 1-\epsilon$, where $m$ denotes the Lebesgue measure on $\hat{\mathbb{C}}$.

All these lemmas follow easily from the following fact:

$$
\{h: \Delta \rightarrow \Delta ; h \text { is holomorphic and proper of degree } \leq d, h(0)=0\}
$$

is a compact set in the topology of uniform convergence on compact sets. We leave the (easy) proofs of this fact and the lemmas to the readers.

2.2. Real rational functions. Let $f$ be a real rational function satisfying the condition (C1).

We shall use $S^{1}$ to denote the extended real line $\hat{\mathbb{R}}=\mathbb{R} \cup\{\infty\}$ and use $\operatorname{Par}(f), C(f)$ to denote the set of parabolic periodic points and the set of critical points of $f$, respectively. All the metrics in this section are the spherical metric on $\hat{\mathbb{C}}$. Let $P(f)$ denote the postcritical set of $f$, that is,

$$
P(f)=\bigcup_{c \in C(f)} \overline{\bigcup_{n=1}^{\infty}\left\{f^{n}(c)\right\} .}
$$

The map $f \mid S^{1}: S^{1} \rightarrow S^{1}$ can be considered as a one-dimensional dynamical system, which has no wandering interval, due to [19].

Proposition 2.1. Let $y \in J(f) \cap S^{1}-\operatorname{Par}(f)$. For any $\epsilon>0$, there is a $\delta>0$ such that for any interval $I \subset S^{1}$ and any $n \in \mathbb{N}$, if $f^{n}(I) \subset B(y, \delta)$, then $|I| \leq \epsilon$.

Proof. Otherwise, there is a positive integer $\epsilon_{0}>0$, and for any $k \in \mathbb{N}$, there is an interval $I_{k} \subset S^{1}$ with $\left|I_{k}\right| \geq \epsilon_{0}$, and a positive integer $n_{k}$ such that

$$
f^{n_{k}}\left(I_{k}\right) \subset B(y, 1 / k) .
$$

After passing to a subsequence, we may assume that there is a non-degenerate interval $I \subset I_{k}$ for all $k$. Then, $\liminf _{n}\left|f^{n}(I)\right|=0$. Thus, there is an attracting or parabolic periodic point $p$ of $f$, such that $\sup _{x \in I} d\left(f^{n}(x), f^{n}(p)\right) \rightarrow 0$ as $n \rightarrow \infty$.

Since $y \in \omega(x)$ for all $x \in I$, we have that $y$ is contained in the orbit of $p$, and so $y$ is an attracting or parabolic periodic point of $f$, which is impossible. 
For any critical point $c$, there is a unique involution $\tau_{c}$ defined by $f\left(\tau_{c}(x)\right)=f(x)$ on a neighbourhood $U_{c}$ in $S^{1}$. We shall fix for any critical point such a neighbourhood $U_{c}$ such that they are pairwise disjoint. An interval $I \subset S^{1}$ is called symmetric if it contains a critical point, say $c$ and if $I \subset U_{c}$ and $\tau_{c}(I)=I$.

For any open interval $I \subset S^{1}$, let $\hat{\mathbb{C}}_{I}$ denote the hyperbolic surface $\hat{\mathbb{C}}-\left(S^{1}-I\right)$. Let $D(I)$ denote the disk such that $D(I) \cap S^{1}=I$ and the boundary $\partial D(I)$ intersects $S^{1}$ orthogonally. The following is an observation of Sullivan, see [29].

LEMMA 2.4. There is a universal constant $\theta>0$ such that $D(I)=\left\{z \in \hat{\mathbb{C}}_{I}: d(z, I)\right.$ $<\theta\}$, where $d$ denotes the hyperbolic distance in $\hat{\mathbb{C}}_{I}$.

Proposition 2.2. Let $f \in \mathcal{F}$. Then either of the following holds:

(i) $\quad J(f)=\hat{\mathbb{C}}$ and $f$ is ergodic; or

(ii) for a.e. $z \in J(f)$, and any $y \in \omega(z)-\operatorname{Par}(f)$, there is a recurrent critical point $c$ such that $\omega(z) \ni c$ and $\omega(c) \ni y$.

Proof. Let $E \subset J(f)$ be a measurable set of positive measure such that $f(E) \subset E(\bmod 0)$ and $m(\hat{\mathbb{C}}-E)>0$. Let $z \in E$ be an arbitrary Lebesgue density point of $E$. Let $y \in \omega(z)$ be an arbitrary point which is not a parabolic periodic point. We claim that there is some recurrent critical point $c$ of $f$ such that $c \in \omega(z)$ and $y \in \omega(c)$.

First of all, we choose a small positive number $\epsilon>0$ so that

(1) for any $c \in C(f)$ which is not recurrent, we have $f^{n}(c) \notin B(c, \epsilon)$ for any $n \in \mathbb{N}$; and

(2) for any $c \in C(f)-\omega(z)$, we have $f^{n}(z) \notin B(c, \epsilon)$ for any $n \in \mathbb{N}$.

Let $\delta>0$ be a small positive number. Let $D_{0}=B(y, \delta / 2) \subset D=B(y, \delta)$ be small disks centred at $y$. There exists a sequence of positive integers $n_{1}<n_{2}<\cdots$ such that $f^{n_{k}}(z) \rightarrow y$ as $k \rightarrow \infty$. Let $A_{k}\left(A_{k}^{0}\right.$, respectively) denote the component of $f^{-n_{k}}(D)$ $\left(f^{-n_{k}}\left(D_{0}\right)\right.$, respectively) containing $z$.

If $f^{n_{k}}: A_{k} \rightarrow D$ has a uniformly bounded degree, then since $z$ is a Lebesgue density point of $E$, by Lemmas 2.2 and 2.3, $m\left(D_{0}-E\right)=0$. Since $E \subset J(f)$, we then have $D_{0} \subset J(f)$. So $f^{n}\left(D_{0}\right)=\hat{\mathbb{C}}$ for some $n \in \mathbb{N}$ and hence $E=\hat{\mathbb{C}}(\bmod 0)$, which is a contradiction. So after passing to a subsequence we may assume that $f^{n_{k}} \mid A_{k}$ has a degree which tends to infinity as $k \rightarrow \infty$. For the same reason, we may assume that $f^{n_{k}} \mid A_{k}^{0}$ also has a large degree.

Thus, there are $0 \leq p_{k}<q_{k}<n_{k}$ such that $f^{p_{k}}\left(A_{k}\right)$ and $f^{q_{k}}\left(A_{k}\right)$ contain the same critical point $c_{k}$ and $f^{n_{k}-p_{k}} \mid f^{p_{k}}\left(A_{k}\right)$ has a uniformly bounded degree. By Lemma 2.1, the hyperbolic diameter of $f^{p_{k}}\left(A_{k}^{0}\right)$ in $f^{p_{k}}\left(A_{k}\right)$ is uniformly bounded from above. By passing to a further subsequence, we may assume that $c_{k}=c$ for all $k$.

If $\delta$ was chosen small, then it follows from Proposition 2.1 that $\max \left\{\left|f^{n}\left(A_{k}\right) \cap S^{1}\right|\right.$ : $\left.p_{k} \leq n \leq n_{k}\right\}<\epsilon$. Since the forward orbit of $c$ enters $f^{q_{k}}\left(A_{k}\right) \cap S^{1}$, which is contained in $B(c, \epsilon)$, it follows from (1) that $c$ is a recurrent critical point. Since $f^{p_{k}}\left(A_{k}^{0}\right) \cap S^{1} \neq \emptyset$, and $f^{p_{k}}\left(A_{k}\right) \subset \hat{\mathbb{C}}_{f^{p_{k}}\left(A_{k}\right) \cap S^{1}}$, and since the hyperbolic diameter of $f^{p_{k}}\left(A_{k}^{0}\right)$ in the hyperbolic surface $f^{p_{k}}\left(A_{k}\right)$ is bounded from above, we have that the spherical diameter of $f^{p_{k}}\left(A_{k}^{0}\right)$ is less than $\epsilon$ (provided that $\delta$ is sufficiently small), which implies that $c \in \omega(z)$ by (2). 
Since $\delta$ can be taken arbitrarily small, the claim follows. The proposition easily follows from the claim.

Let $C_{r}(f) \subset C(f)$ denote the set of non-periodic recurrent critical points. For any $c, d \in C_{r}(f)$, we say $c \sim d$ if $\omega(c) \ni d$ and $\omega(d) \ni c$. An order is defined on the set of equivalence classes as follows: $[c] \prec[d]$ if $\omega(c) \ni d$ but $\omega(d) \not \supset c$. Let

$$
J_{c}=\{z \in J(f): \omega(z) \ni c \text { but } \omega(z) \not \supset d \text { for any }[d] \prec[c]\} .
$$

It follows from Proposition 2.2 that either $J(f)=\hat{\mathbb{C}}$ and $f$ is ergodic or

$$
J(f) \subset \bigcup_{c \in C_{r}(f)} J_{c}(\bmod 0) .
$$

\section{Invariant line fields}

Recall that $a$ line field $\mu$ is a measurable Beltrami differential on $\hat{\mathbb{C}}$ such that $|\mu|=1$ on a set $E$ of positive measure and $\mu$ vanishes elsewhere. The set $E$ is the support of $\mu$. A line field is holomorphic on an open set $U$ if $\mu=\bar{\phi} /|\phi|$ a.e. for $\phi$ a holomorphic quadratic differential on $U$. A line field $\mu$ is called (f-)invariant if $f^{*}(\mu)=\mu$ a.e. We say that $f$ carries an invariant line field on the Julia set if there is an $f$-invariant line field $\mu$ with a support contained in its Julia set (up to a set of measure zero).

A rational map $f$ is called a Lattés example if it is doubly covered by an integral torus endomorphism. Such a rational map carries an invariant line field on its Julia set, which is $\hat{\mathbb{C}}$. It is a conjecture that Lattés examples are the only rational maps which carry an invariant line field on the Julia set, see $[\mathbf{1 8}, \mathbf{2 2}]$.

It is useful to remember the following, which is Lemma 3.16 in [20].

LEMMA 3.1. Let $\mu$ be an $f$-invariant line field which is holomorphic on a non-empty open set contained in the Julia set. Then $f$ is a Lattés example.

Proposition 3.1. Let $f \in \mathcal{F}$. Assume that $f$ carries an invariant line field $\mu$ with support $E \subset J(f)$. Then either

(i) for a.e. $z \in E$ and for any $y \in \omega(z)$ which is not a parabolic periodic point, there exists $c \in C_{r}(f)$ such that $c \in \omega(z)$ and $\omega(c) \ni y$; or

(ii) $f$ is a Lattés example.

Proof. Take a Lebesgue density point $z$ of $E$ and let $y \in \omega(z)-\operatorname{Par}(f)$. If there is no $c \in C_{r}(f) \cap \omega(z)$ such that $\omega(c) \ni y$, then by the same argument as that in the proof of Proposition 2.2, we will obtain a sequence of proper mappings $f^{n_{k}}: A_{k} \rightarrow B(y, \delta)$ for some $\delta>0$ such that $x \in A_{k}$ and $f^{n_{k}} \mid A_{k}$ has a uniformly bounded degree, and $f^{n_{k}}(x) \rightarrow y$ as $k \rightarrow \infty$. It then easily follows that $\mu$ is holomorphic on some non-empty open set and hence $f$ is doubly covered by an integral torus endomorphism.

We shall give more criteria for the non-existence of invariant line fields. The idea is taken from [20] and [21]: Uniformly nonlinearity implies the absence of invariant line fields. 
An invariant line field $\mu$ is called almost continuous at a point $x$ if for any $\epsilon>0$,

$$
\frac{m(\{z \in B(x, r):|\mu(z)-\mu(x)| \geq \epsilon\})}{m(B(x, r))} \rightarrow 0
$$

as $r \rightarrow 0$, where $\mu=\mu(z) d \bar{z} / d z$ on a conformal coordinate around $x$. Note that the definition does not depend on the choice of the coordinate.

Given a rational function $f$, consider the collection $\mathcal{H}(f)$ of all holomorphic maps $h: U \rightarrow V$, where $U, V$ are open sets such that there exist $i, j \in \mathbb{N}$ such that $f^{i} \circ h=f^{j}$ on $U$. Obviously for any element $h: U \rightarrow V$ in $\mathcal{H}(f), h^{*}(\mu \mid V)=\mu \mid U$, i.e.

$$
\mu(z)=\mu(h(z)) \overline{h^{\prime}(z)} / h^{\prime}(z) \text { a.e. on } U .
$$

PROPOSITION 3.2. Let $f$ be a rational function of degree $\geq 2$ and $x$ be a point in $J(f)$. If there is a positive constant $C>1$ and a positive integer $N \geq 2$ and a sequence $h_{n}: U_{n} \rightarrow V_{n}$ of elements of $\mathcal{H}(f)$ with the following properties:

(i) $U_{n}, V_{n}$ are topological disks and

$$
\operatorname{diam}\left(U_{n}\right) \rightarrow 0, \operatorname{diam}\left(V_{n}\right) \rightarrow 0
$$

as $n \rightarrow \infty$;

(ii) $h_{n}$ is a proper map with degree between 2 and $N$;

(iii) for some $u \in U_{n}$ such that $h_{n}^{\prime}(u)=0$ and for $v=h_{n}(u)$ we have

$$
\max _{z \in \partial U_{n}} d(z, u) \leq C d\left(u, \partial U_{n}\right)
$$

and

$$
\max _{z \in \partial V_{n}} d(z, v) \leq C d\left(v, \partial V_{n}\right)
$$

(iv)

$$
d\left(U_{n}, x\right) \leq C \operatorname{diam}\left(U_{n}\right), \quad d\left(V_{n}, x\right) \leq C \operatorname{diam}\left(V_{n}\right),
$$

where diam and, $d$ denote the diameter and the distance in the spherical metric respectively.

Then for any $f$-invariant line field $\mu, x \notin \operatorname{supp}(\mu)$ or $\mu$ is not almost continuous at $x$.

Proof. By changing the coordinates using a Möbius transformation, we can assume that $x \in \mathbb{C}$. The conditions in (i), (iii) and (iv) also hold for the Euclidean metric by changing the constant $C$. Here, all distances, diameters and areas are measured in the Euclidean metric on $\mathbb{C}$ unless otherwise stated.

Fix any $u_{n} \in U_{n}$ such that $h_{n}^{\prime}\left(u_{n}\right)=0$ and let $v_{n}=h_{n}\left(u_{n}\right)$. Denote by $\alpha_{n}$ and $\beta_{n}$ the linear transformation of $\mathbb{C}$ such that $\alpha_{n}^{\prime}>0, \beta_{n}^{\prime}>0$ and

$$
\alpha_{n}\left(u_{n}\right)=\beta_{n}\left(v_{n}\right)=0, \quad \operatorname{diam}\left(\alpha_{n} U_{n}\right)=\operatorname{diam}\left(\beta_{n} V_{n}\right)=1 .
$$

Denote $X_{n}=\alpha_{n} U_{n}$ and $Y_{n}=\beta_{n} V_{n}$. And denote by $H_{n}$ the function $\beta_{n} \circ h_{n} \circ \alpha_{n}^{-1}$ : $X_{n} \rightarrow Y_{n}$. By condition (iii), we have

$$
\begin{gathered}
\min _{z \in \partial X_{n}}|z| \geq \max _{z \in \partial X_{n}}|z| / C \geq 1 /(2 C), \\
\min _{z \in \partial Y_{n}}|z| \geq \max _{z \in \partial Y_{n}}|z| / C \geq 1 /(2 C) .
\end{gathered}
$$


Thus $X_{n}, Y_{n} \supset B(0,1 /(2 C))$. So after passing to a subsequence we may assume that $\left(X_{n}, 0\right) \rightarrow(X, 0)$ and $\left(Y_{n}, 0\right) \rightarrow(Y, 0)$ as $n \rightarrow \infty$ in the Caratheodory topology for some topological disk $X, Y$ and there is a holomorphic function $H:(X, 0) \rightarrow(Y, 0)$ such that for any compact set $K$ in $X, H_{n}|K \rightarrow H| K$ uniformly as $n \rightarrow \infty$.

\section{Claim. The function $H$ is not a constant function.}

Indeed, since $X_{n} \subset B(0,1)$, for some $\delta=\delta(C)>0$, in the hyperbolic surface $X_{n}$, the hyperbolic ball $B_{X_{n}}(0, \delta)$ is contained in the Euclidean ball $B(0,1 /(4 C))$. By Lemma 2.1, there is a constant $\epsilon>0$ which depends only on $C$ and $N$, such that $H_{n}(B(0,1 /(4 C)))$ contains the hyperbolic ball $B_{Y_{n}}(0, \epsilon)$ in the hyperbolic surface $Y_{n}$. Since $Y_{n} \supset$ $B(0,1 /(2 C)), B_{Y_{n}}(0, \epsilon) \supset B\left(0, \epsilon^{\prime}\right)$ for some $\epsilon^{\prime}>0$. For any $n \in \mathbb{N}$, let $z_{n} \in B(0,1 /(4 C))$ be a point such that $\left|H_{n}\left(z_{n}\right)\right|=\epsilon^{\prime}$. After passing to a further subsequence, we may assume that $z_{n} \rightarrow z$ as $n \rightarrow \infty$. Thus $|H(z)|=\epsilon^{\prime}$. Note $H(0)=0$, and hence the claim follows.

Consequently, $H$ is a proper map of degree between 2 and $N$.

Assume now that $|\mu(x)|=1$ and that $\mu$ is almost continuous at $x$. Without loss of generality we can assume that $\mu(x)=1$. Let us prove that for any $\delta>0$, we have

$$
\frac{m\left(\left\{z \in U_{n}:|\mu(z)-1| \geq \delta\right\}\right)}{m\left(U_{n}\right)} \rightarrow 0,
$$

as $n \rightarrow \infty$.

Let $r_{n}=\max _{z \in U_{n}} d(x, z)$ and $s_{n}=d\left(u_{n}, \partial U_{n}\right)$. Then $r_{n} \rightarrow 0$ as $n \rightarrow \infty$. Thus, by the definition of almost continuity, we have

$$
\frac{m\left(\left\{z \in B\left(x, r_{n}\right):|\mu(z)-1| \geq \delta\right\}\right)}{m\left(B\left(x, r_{n}\right)\right)} \rightarrow 0 .
$$

Note that by (iii) and (iv), we have

$$
m\left(B\left(x, r_{n}\right)\right) \asymp r_{n}^{2} \asymp s_{n}^{2} \asymp m\left(U_{n}\right) .
$$

Thus, statement (1) holds.

Similarly, we can prove that for any $\delta>0$,

$$
\frac{m\left(\left\{z \in V_{n}:|\mu(z)-1| \geq \delta\right\}\right)}{m\left(V_{n}\right)} \rightarrow 0,
$$

as $n \rightarrow \infty$

Let $\mu_{n}=\left(\alpha_{n}^{-1}\right)^{*}\left(\mu \mid U_{n}\right)$ be a Beltrami differential on $X_{n}$ and $v_{n}=\left(\beta_{n}^{-1}\right)^{*}\left(\mu \mid V_{n}\right)$ be a Beltrami differential on $Y_{n}$. Obviously $H_{n}^{*} v_{n}=\mu_{n}$. It follows from (1) and (2) that for any $\delta>0$

$$
m\left(\left\{z \in X_{n}:\left|\mu_{n}(z)-1\right| \geq \delta\right\}\right) \rightarrow 0
$$

and

$$
m\left(\left\{z \in Y_{n}:\left|v_{n}(z)-1\right| \geq \delta\right\}\right) \rightarrow 0
$$

as $n \rightarrow \infty$

Let $L$ be a small Euclidean ball whose closure is contained in $X$ such that $H$ is univalent on a neighbourhood of $\bar{L}$. Let $\eta>0$ be a constant such that $\left|H^{\prime}(z)\right| \geq 2 \eta$ for any $z \in L$. 
Since $H_{n}|L \rightarrow H| L$ uniformly, we have that for $n$ sufficiently large, $H_{n} \mid L$ is univalent and also $\left|H_{n}^{\prime}(z)\right| \geq \eta$ on $L$.

By (3) and (4), for any $\delta>0$, we have

$$
m\left\{z \in L:\left|\mu_{n}(z)-1\right| \geq \delta\right\} \rightarrow 0
$$

and

$$
m\left\{w \in H_{n}(L):\left|v_{n}(z)-1\right| \geq \delta\right\} \rightarrow 0
$$

as $n \rightarrow \infty$. Note that

$$
m\left\{z \in L:\left|v_{n}\left(H_{n}(z)\right)-1\right| \geq \delta\right\} \leq \eta^{-2} m\left\{w \in H_{n}(L):\left|v_{n}(w)-1\right| \geq \delta\right\} \rightarrow 0
$$

as $n \rightarrow \infty$.

For any $\delta \in(0,1)$, let

$$
A_{n}(\delta)=\left\{z \in L:\left|\overline{\frac{H_{n}^{\prime}(z)}{H_{n}^{\prime}(z)}}-1\right| \geq \delta\right\}
$$

as $n \rightarrow \infty$. For a.e. $z \in A_{n}(\delta)$, we have $\mu_{n}(z)=v_{n}\left(H_{n}(z)\right) \overline{H_{n}^{\prime}(z)} / H_{n}^{\prime}(z)$. Thus, for such a $z$, either $\left|v_{n}\left(H_{n}(z)\right)-1\right|>\delta / 10$, or $\left|v_{n}(z)-1\right|>\delta / 10$. Thus, we have

$$
m\left(A_{n}(\delta)\right) \rightarrow 0
$$

as $n \rightarrow \infty$. Consequently, we have that, for any $\delta>0$,

$$
m\left\{z \in L:\left|\frac{\overline{H^{\prime}(z)}}{H^{\prime}(z)}-1\right|>\delta\right\}=0 .
$$

Since $\delta$ can be taken to be arbitrarily small, we know that for a.e. $z \in L, \overline{H^{\prime}(z)}=H^{\prime}(z)$. But then $H^{\prime}$ is a constant function, and hence it is equal to 0 , i.e. $H$ is a constant function, which is a contradiction.

To construct the family $\left\{h_{n}\right\}$ we usually first construct a sequence of restrictions of forward iterates of $f$, then try to pull them back to the neighbourhood of $x$.

For any annulus $A$ with a finite modulus, we define the core curve of $A$ to be the unique simple geodesic in $A$. In other words, if $\phi: A \rightarrow\{z \in \mathbb{C}: 1<|z|<R\}$ is a conformal map, then the core curve is $\phi^{-1}(\{z \in \mathbb{C}:|z|=\sqrt{R}\})$. For any topological disk $U$, and any compact set $K \subset U$, we use $\bmod (K, U)$ to denote the supremum of the modulus of an annulus which is contained in $U$ and surrounds $K$.

COROLLARY 3.3. Let $f$ be a rational function of degree $d \geq 2$ and $x$ be a point in the Julia set $J(f)$. If there exists a positive constant $\delta$, a positive integer $N \geq 2$, sequences $\left\{s_{n}\right\},\left\{p_{n}\right\},\left\{q_{n}\right\}$ of positive integers, sequences $\left\{A_{n}\right\},\left\{B_{n}\right\}$ of topological disks with the following properties:

(i) $\quad f^{s_{n}}: A_{n} \rightarrow B_{n}$ is a proper map whose degree is between 2 and $N$;

(ii) $\operatorname{diam}\left(B_{n}\right) \rightarrow 0$ as $n \rightarrow \infty$;

(iii) $f^{p_{n}}(x) \in A_{n}, f^{q_{n}}(x) \in B_{n}$ and

$$
\bmod \left(\left\{f^{p_{n}}(u): u \in A_{n},\left(f^{p_{n}}\right)^{\prime}(u)=0\right\} \cup\left\{f^{p_{n}+s_{n}}(x), f^{q_{n}}(x)\right\}, B_{n}\right)>\delta ;
$$


(iv) for any $n \in \mathbb{N}$, there exists $g_{n}^{A}: A_{n} \rightarrow \mathbb{C}$ and $g_{n}^{B}: B_{n} \rightarrow \mathbb{C}$ such that

$$
f^{p_{n}} \circ g_{n}^{A}=i d_{A_{n}}, \quad g_{n}^{A}\left(f_{n}^{p}(x)\right)=x
$$

and

$$
f^{q_{n}} \circ g_{n}^{B}=i d_{B_{n}}, \quad g_{n}^{B}\left(f^{q_{n}}(x)\right)=x ;
$$

(v) $s_{n} \rightarrow \infty, p_{n} \rightarrow \infty, q_{n} \rightarrow \infty$ as $n \rightarrow \infty$;

then for any $f$-invariant line field $\mu, \mu(x)=0$ or $\mu$ is not almost continuous at $x$.

Proof. By condition (iii) there is an annulus $\Omega_{n} \subset B_{n}$ surrounding $\left\{f^{p_{n}}(u): u \in A_{n}\right.$, $\left.\left(f^{p_{n}}\right)^{\prime}(u)=0\right\} \cup\left\{f^{p_{n}+s_{n}}(x), f^{q_{n}}(x)\right\}$ such that $\bmod \left(\Omega_{n}\right)=\delta$. Let $\gamma_{n}$ be the core curve of $\Omega_{n}$ and $B_{n}^{\prime}$ be the topological disk bounded by this curve. There is a constant $C(\delta)>1$ such that for any $b \in\left\{f^{s_{n}}(u):\left(f^{s_{n}}\right)^{\prime}(u)=0\right\} \cup\left\{f^{p_{n}+s_{n}}(x), f^{q_{n}}(x)\right\}$,

$$
\max _{z \in \gamma_{n}} d(z, b) \leq C(\delta) d\left(b, \gamma_{n}\right) \text {. }
$$

Let $A_{n}^{\prime}$ be the component of $f^{-s_{n}}\left(B_{n}^{\prime}\right)$ contained in $A_{n}$. Let $U_{n}=g_{n}^{A} A_{n}^{\prime}, V_{n}=g_{n}^{B} B_{n}^{\prime}$ and

$$
h_{n}=g_{n}^{B} \circ f^{s_{n}} \circ g_{n}^{A}: U_{n} \rightarrow V_{n} .
$$

Then $\left\{h_{n}: U_{n} \rightarrow V_{n}\right\}$ is a sequence satisfying the hypothesis of Proposition 3.2.

\section{Quasi-polynomial-like mappings}

In this section, we recall two notions: one is 'a real box mapping' which we used in [26]; and the other is 'a quasi-polynomial-like mapping', used by Levin and van Strien in [12], which turns out to be the complex counterpart of the first notion.

Fix an $f \in \mathcal{F}$. A (real) maximal chain is a sequence of open intervals $\left\{G_{i}\right\}_{i=0}^{n}$ such that $f\left(G_{i}\right) \subset G_{i+1}$ and $G_{i}$ is the maximal interval with this property, for any $0 \leq i \leq n-1$. The order is the number of $G_{i}$ s containing a critical point. If $\left\{G_{i}\right\}_{i=0}^{n}$ is a maximal chain we shall say that $G_{0}$ is a pull-back of $G_{n}$. If $G_{i}$ does not contain a critical point for any $0 \leq i \leq n-1$, then the chain is called monotone and $G_{0}$ is called a monotone pull-back of $G_{n}$. If $G_{i}$ does not contain a critical point for any $0<i<n$ but $G_{0}$ contains exactly one, we shall say that the chain is unimodal, and $G_{0}$ is a unimodal pull-back of $G_{n}$.

Recall that an open interval $T$ is called nice if $f^{n}(\partial T) \cap T=\emptyset$ for any $n \in \mathbb{N}$. Clearly, if $G$ and $G^{\prime}$ are two pull-backs of $T$ then either they are disjoint or one is contained in the other. We call an interval $T$ properly periodic if there is a positive integer $s \geq 1$, such that the interiors of $T, f(T), \ldots, f^{s-1}(T)$ are pairwise disjoint and $f^{s}(T) \subset T$, $f^{s}(\partial T) \subset \partial T$. In this case $s$ is called the period of $T$ and $f^{s}: T \rightarrow T$ will be called $a$ renormalization of $f$. Let us say that $f$ is renormalizable at $c$ if there is a properly periodic interval containing $c$ and that $f$ is infinitely renormalizable at $c$ if there are a sequence of properly periodic intervals $\left\{T_{n}\right\}$ containing $c$ with periods $s_{n} \rightarrow \infty$ as $n \rightarrow \infty$. If $f$ is not infinitely renormalizable at $c$, we shall say that $f$ is only finitely renormalizable at $c$.

Let $c \in C_{r}(f)$ and let $c_{1}, c_{2}, \ldots, c_{b}$ be the critical points in $[c]$. Let $I_{i} \ni c_{i}$ be small nice intervals, $i=1,2, \ldots, b$. Let $J_{i}^{j}, 0 \leq j<r_{i}+1$ be pairwise disjoint intervals contained in $I_{i}$ such that $J_{i}^{0} \ni c_{i}$, where $r_{i} \in \mathbb{N} \cup\{0, \infty\}$. A mapping

$$
B: \bigcup_{i=1}^{b} \bigcup_{j=0}^{r_{i}} J_{i}^{j} \rightarrow \bigcup_{i=1}^{b} I_{i}
$$


is called a (real) box mapping (induced by $f$ ) corresponding to $c$ if for each $1 \leq i \leq b$ and each $0 \leq j<r_{i}+1$ there is a $k(i, j) \in\{1,2, \ldots, b\}$ and $p(i, j) \in \mathbb{N}$ with the following properties:

(B1) $B\left|J_{i}^{j}=f^{p_{i, j}}\right| J_{i}^{j}$;

(B2) there is a maximal chain $\left\{G_{k}\right\}_{k=0}^{p_{i, j}}$ with $G_{p_{i, j}}=I_{k_{i, j}}$ and $G_{0}=J_{i}^{j}$, moreover the chain is unimodal if $j=0$ and monotone otherwise;

(B3) $J_{i}^{j} \cap \operatorname{orb}([c]) \neq \emptyset$;

(B4) $\operatorname{orb}([c]) \cap I_{i} \subset \bigcup_{j=0}^{r_{i}} J_{i}^{j}$;

(B5) for any $x \in \operatorname{orb}([c]) \cap J_{i}^{j}$, we have $f(x), f^{2}(x), \ldots, f^{p_{i, j}-1}(x) \notin \bigcup_{i=1}^{b} I_{i}$.

Remark 4.1. In the language of [26], (5) is a box mapping of type II which is the first return map to its image.

The following is a natural way to construct box mappings. For any small nice interval $I \ni c$ and any $c^{\prime} \in[c], c^{\prime} \neq c$, let $I\left(c^{\prime}\right)$ be the component containing $c^{\prime}$ of the domain of the first return map (of $f$ ) to $I$. Denote these intervals $I\left(c^{\prime}\right)$ by $I_{2}, I_{3}, \ldots, I_{b}$ and put $I_{1}=I$. For any $2 \leq i \leq b$ let $r_{i}=0$ and let $J_{i}^{0}=I_{i}$. For any $x \in \operatorname{orb}([c]) \cap I$, let $J(x)$ denote the component of the domain of the first return map to $I$ containing $x$. Let $r_{1}+1$ be the number of these intervals $J(x)$ and let $J_{1}^{0} \ni c, J_{1}^{1}, \ldots$ be these intervals. Finally define $B_{I}: \bigcup_{i=1}^{b} \bigcup_{j=0}^{r_{i}} J_{i}^{j} \rightarrow \bigcup_{i=1}^{b} I_{i}$ to be the first return map of $f$ to $\bigcup_{i=1}^{b} I_{i}$. We shall call $B_{I}$ the box mapping associated to $I$. Note that if $\omega(c)$ is a minimal set, then $r_{1}$ is finite.

Let $V_{i}$, be topological disks containing $c_{i}, i=1,2, \ldots, b$, such that $V_{i_{1}} \cap V_{i_{2}} \cap$ $\operatorname{orb}([c])=\varnothing$ for any $1 \leq i_{1}<i_{2} \leq b$. For each $1 \leq i \leq b$, let $U_{i}^{j}$ be topological disks contained in $V_{i}, 0 \leq j<r_{i}+1$, for some $r_{i} \in \mathbb{N} \cup\{0, \infty\}$ such that for any $0 \leq j_{1}<j_{2}<r_{i}+1$, we have $U_{i}^{j_{1}} \cap U_{i}^{j_{2}} \cap \operatorname{orb}([c])=\emptyset$.

A collection $\left\{F_{i, j}: U_{i}^{j} \rightarrow \bigcup_{i=1}^{b} V_{i} \mid 1 \leq i \leq b, 0 \leq j<r_{i}+1\right\}$ is called a quasipolynomial-like mapping (induced by $f$ ) corresponding to $c$ if the following holds. For any $1 \leq i \leq b$ and any $0 \leq j<r_{i}+1$, we have $1 \leq k_{i, j} \leq b$ and $p_{i, j} \in \mathbb{N}$, such that:

(H1) $F_{i, j}=f^{p_{i, j}} \mid U_{i}^{j}$;

(H2) $F_{i, j}\left(U_{i}^{j}\right)=V_{k_{i, j}}$ and the map $F_{i, j}$ is a branched covering with a unique critical point $c_{i}$ for $j=0$ and is conformal otherwise;

(H3) for any $1 \leq i \leq b$ and $0 \leq j<r_{i}+1$, orb $([c]) \cap U_{i}^{j} \neq \emptyset$;

(H4) for any $1 \leq i \leq b$, we have orb $([c]) \cap V_{i} \subset \operatorname{orb}([c]) \cap\left(\bigcup_{j=0}^{r_{i}} U_{i}^{j}\right)$;

(H5) for any $1 \leq i \leq b$ and $0 \leq j<r_{i}+1$ and any $x \in \operatorname{orb}([c]) \cap U_{i}^{j}$, if $F_{i, j}=f^{p}$, then $f(x), f^{2}(x), \ldots, f^{p-1}(x) \notin \bigcup_{i=1}^{b} V_{i}$.

Remark. Quasi-polynomial-like mappings are not mappings in the classical sense. They naturally appear as appropriate holomorphic extensions of real box mappings. We allow intersection of these domains $U_{i}^{j}$ in order to obtain an advantage in constructing extensions. Note that we even allow the real traces of $U_{i}^{j}$ to intersect each other, which was excluded in [12]. 
A quasi-polynomial-like mapping $\mathbf{F}=\left\{F_{i, j}\right\}$ determines a map

$$
F: \bigcup_{i, j}\left(U_{i}^{j}-\bigcup_{\left(i^{\prime}, j^{\prime}\right) \neq(i, j)} U_{i^{\prime}}^{j^{\prime}}\right) \rightarrow \bigcup_{i} V_{i}
$$

in a natural way. Let $K(\mathbf{F})$ be the set of points $z$ such that $F^{n}(z)$ is well defined for any $n \in \mathbb{N}$. We shall call $K(\mathbf{F})$ the Julia set of $\left\{F_{i, j}\right\}$, which is always a measurable set. By the definition, orb $([c]) \cap\left(\bigcup_{i} V_{i}\right)$ belongs to the set $K(\mathbf{F})$.

For any $x \in K(\mathbf{F})$, and any $n \geq 0$, there is a unique $\left(i_{n}, j_{n}\right)$ such that $F^{n}(x) \in U_{i_{n}}^{j_{n}}$. So for any topological disk $F^{n}(x) \in A \subset V_{i_{n}}$, we can consider the pull-back of $A$ under $\mathbf{F}$ along the orbit $x, F(x), \ldots, F^{n}(x)$, which is defined to be the topological disk $P$ containing $x$, such that $F_{i_{m-1}, j_{m-1}} \circ F_{i_{m-2}, j_{m-2}} \circ \cdots \circ F_{i_{0}, j_{0}}(P) \subset U_{i_{m}}^{j_{m}}$ for all $0 \leq m<n$ and $F_{i_{n-1}, j_{n-1}} \circ F_{i_{n-2}, j_{n-2}} \circ \cdots \circ F_{i_{0}, j_{0}}(P)=A$. Note that such a topological disk $P$ always exists. We shall write $P=\operatorname{Comp}_{x}(\mathbf{F}, n, A)$ and sometimes we will use $F^{n}: P \rightarrow A$ to denote the map $F_{i_{n-1}, j_{n-1}} \circ \cdots \circ F_{i_{0}, j_{0}} \mid P$. Similarly, we shall use $V(\mathbf{F}, x)$ to denote the topological disk $V_{i}$ containing $x$, and $U(\mathbf{F}, x)$ the topological disk $U_{i}^{j}$ containing $x$. We shall sometimes omit the quasi-polynomial-like mapping $\mathbf{F}$ in these notations, provided that it is clear from the context which $\mathbf{F}$ is referred to.

LEMMA 4.1. If $0 \leq m<n$ are two integers and $x \in K(\mathbf{F})$, then we have

$$
\operatorname{Comp}_{x}\left(n, V\left(F^{n}(x)\right)\right) \subset \operatorname{Comp}_{x}\left(m, V\left(F^{m}(x)\right)\right) .
$$

Given a real box mapping as in (5), we can construct a quasi-polynomial-like mapping in the following way. For any $1 \leq i \leq b$, let $V_{i}=\hat{\mathbb{C}}_{I_{i}}$. For any $0 \leq j<r_{i}+1$, let $U_{i}^{j}$ be the component of $f^{-p_{i, j}}\left(V_{i}\right)$ containing $J_{i}^{j}$, where $p_{i, j}$ is as in (B1). Let $F_{i, j}=f^{p_{i, j}} \mid U_{i}^{j}$. Then $\left\{F_{i, j}\right\}$ is a quasi-polynomial-like mapping. This construction can always be done but the extended map may not possess any geometric property.

5. The measurable dynamics of $f \mid J_{c}$ for a recurrent critical point $c$ with a non-minimal $\omega$-limit set

The purpose of this section is to consider the dynamics of $f \mid J_{c}$ for a recurrent critical point $c$ such that $\omega(c)$ is not minimal. ( $J_{c}$ is defined at the end of $\S 2$.) The result is as follows.

THEOREM 5.1. Let $f \in \mathcal{F}$, and $c \in C_{r}(f)$ be such that $\omega(c)$ is not minimal. Then

(i) $m\left(J_{c}\right)=0$ or $J(f)=\hat{\mathbb{C}}$ and $f$ is ergodic; and

(ii) $f$ carries no invariant line field on $J_{c}$.

We shall use the real bound developed in [26] to construct a quasi-polynomial-like mapping corresponding to $c$. Theorem 5.1 will be shown by a combinatorial argument.

The following is Proposition 6.2 in [26].

Proposition 5.2. For any $\rho>0$ and any $\epsilon>0$, there is a real box mapping $B: \bigcup_{i=1}^{b} \bigcup_{j=0}^{r_{i}} J_{i}^{j} \rightarrow \bigcup_{i=1}^{b} I_{i}$ corresponding to $c$ such that $\max _{i=1}^{b}\left|I_{i}\right| \leq \epsilon$; and for any $1 \leq i \leq b$, there exists a symmetric interval $T_{i}$ containing the $\rho$-neighbourhood of $I_{i}$, with the following properties: 
(i) if $B \mid J_{i}^{j}=f^{p}$ and $B\left(J_{i}^{j}\right) \subset I_{k}$, then the maximal chain $\left\{G_{n}\right\}_{n=1}^{p}$ with $G_{p}=T_{k}$ and $G_{1} \supset f\left(J_{i}^{j}\right)$ is a monotone chain; and

(ii) if $G_{0}$ is the component of $f^{-1}\left(G_{1}\right)$ containing $J_{i}^{j}$, then $J_{i}^{j} \subset T_{i}$; moreover,

(iii) if $J_{i}^{j} \neq I_{i}$, then $G_{0} \subset I_{i}$.

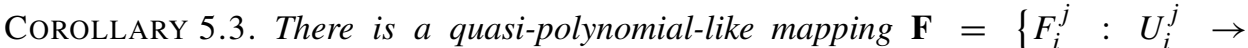
$\left.\bigcup_{i=1}^{b} V_{i} \mid 1 \leq i \leq b, 0 \leq j<r_{i}+1\right\}$ corresponding to $c$, such that $\sum_{i=1}^{b} r_{i}=\infty$ and for some $C>0$ and $N \in \mathbb{N}$, we have:

(i) for each $1 \leq i \leq b$, there is a topological disk $W_{i} \supset \supset V_{i}$, such that $\bmod \left(W_{i}-\overline{V_{i}}\right)$ $\geq C$

(ii) for any $1 \leq i \leq b$ and $0 \leq j<r_{i}+1$, if $F_{i, j}=f^{p_{i, j}}$ and $F_{i, j}\left(U_{i}^{j}\right)=V_{k}$, then there is a topological disk $X_{i}^{j} \supset U_{i}^{j}$ such that $f^{p_{i, j}}: X_{i}^{j} \rightarrow W_{k}$ is a proper map of degree $\leq N$. Moreover, if $U_{i}^{j} \neq V_{i}$, then $X_{i}^{j} \subset V_{i}$.

Proof. Let $B: \bigcup_{i=1}^{b} \bigcup_{j=0}^{r_{i}} J_{i}^{j} \rightarrow \bigcup_{i=1}^{b} I_{i}$ be a real box mapping satisfying the requirements in Proposition 5.2 for a large $\rho$ and small $\epsilon$. Since $\omega(c)$ is not minimal, $\sum_{i} r_{i}=\infty$ if $\epsilon$ is sufficiently small. We shall extend this box mapping to a quasipolynomial-like mapping. To fix the notation, we assume that $J_{i}^{0}=I_{i}$ if and only if $b^{\prime}+1 \leq i \leq b$ for some $1 \leq i \leq b^{\prime}$. By possibly interchanging the indices, we can always assume this to be so. For each $1 \leq i \leq b$, let $S_{i} \supset I_{i}$ be the symmetric interval such that $T_{i}$ is the $\rho / 2$-neighbourhood of $S_{i}$ and let $W_{i}=D\left(S_{i}\right)$. For any $1 \leq i \leq b^{\prime}$, define $V_{i}=D\left(I_{i}\right)$.

For any $b^{\prime}+1 \leq i \leq b$, let $s=s_{i}$ be the minimal positive integer such that $B^{s_{i}}\left(I_{i}\right) \subset I_{i^{\prime}}$ for some $1 \leq i^{\prime} \leq b^{\prime}$. Such a positive integer always exists and is no more than $b-b^{\prime}$. If $B^{s_{i}}\left|I_{i}=f^{k_{i}}\right| I_{i}$, then let $V_{i}$ be the component of $f^{-k_{i}}\left(D\left(I_{i^{\prime}}\right)\right)$ containing $I_{i}$. Let $\tilde{V}_{i} \supset V_{i}$ be a component of $f^{-k_{i}}\left(D\left(T_{i^{\prime}}\right)\right)$, then $f^{k_{i}}: \tilde{V}_{i} \rightarrow D_{T_{i^{\prime}}}$ is a proper map of degree uniformly bounded from above. Since $V_{i^{\prime}}$ has a small diameter in the hyperbolic Riemann surface $W_{i^{\prime}}$, $V_{i}$ has a small diameter in the hyperbolic Riemann surface $\tilde{V}_{i}$. Thus $V_{i}$ is compactly contained in $W_{i}$ and the annulus $W_{i}-\overline{V_{i}}$ has a large modulus.

For any $1 \leq i \leq b$ and $0 \leq j<r_{i}+1$, let $m=m(i, j)$ be such that $B\left(J_{i}^{j}\right) \subset I_{m}$ and let $U_{i}^{j}\left(X_{i}^{j}, Y_{i}^{j}\right.$, respectively) be the component of $f^{-p_{i, j}}\left(V_{m}\right)\left(f^{-p_{i, j}}\left(W_{m}\right), f^{-p_{i, j}}\left(\hat{\mathbb{C}}_{T_{m}}\right)\right.$, respectively) containing $J_{i}^{j}$, where $p_{i, j} \in \mathbb{N}$ is such that $B\left|J_{i}^{j}=f^{p_{i, j}}\right| J_{i}^{j}$. Then $f^{p_{i, j}}$ : $Y_{i}^{j} \rightarrow \hat{\mathbb{C}}_{T_{m}}$ is a proper map of a degree uniformly bounded from above. Since $W_{m}$ has a small diameter in the hyperbolic Riemann surface $\hat{\mathbb{C}}_{T_{m}}$, by Lemma $2.1, X_{i}^{j}$ has a small diameter in the hyperbolic Riemann surface $Y_{i}^{j}$. Thus $X_{i}^{j} \subset V_{i}$ if $1 \leq i \leq b^{\prime}$, since in that case, the real trace of $Y_{i}^{j}$ is contained in $I_{i}$.

Define $F_{i, j}\left|U_{i}^{j}=f^{p_{i, j}}\right| U_{i}^{j}$. Then $\left\{F_{i, j}\right\}$ becomes a quasi-polynomial-like mapping as desired.

Proposition 5.4. Assume that $\omega(c)$ is not minimal. Then for some $N \in \mathbb{N}$, and for any $x \in J_{c}$, there are some $d \in[c]$ and some neighbourhoods $U \subset \subset U^{\prime}$ of $d$ with the following property: for infinitely many positive integers $n$, there is a neighbourhood $P$ of $x$ such that $f^{n}: P \rightarrow U^{\prime}$ is a proper map of degree $\leq N$, and $f^{n}(x) \in U$. 
Proof. Let $\mathbf{F}=\left\{F_{i, j}: U_{i}^{j} \rightarrow \bigcup_{i=1}^{b} V_{i} \mid 1 \leq i \leq b, 0 \leq j<r_{i}+1\right\}$ be the quasipolynomial-like mapping as in the previous proposition. The real trace of $\mathbf{F}$ is a real box mapping. Let $p_{i, j}, k_{i, j} \in \mathbb{N}$ be such that $F_{i, j}=f^{p_{i, j}} \mid U_{i}^{j}, F_{i, j}\left(U_{i}^{j}\right) \subset V_{k_{i, j}}$. We may assume that $r_{1}=\infty$ and that for some $1 \leq b^{\prime} \leq b, U_{i}^{0}=V_{i}$ if and only if $b^{\prime}+1 \leq i \leq b$. Let $F$ be the map determined by $\mathbf{F}$ as described at the top of page 967 .

For any $1 \leq i \leq b$, let $t_{i}$ be the minimal non-negative number such that

$$
F^{t_{i}}\left(c_{i}\right) \subset \bigcup_{i=1}^{b^{\prime}} V_{i},
$$

and let $s_{i}$ be the non-negative integer such that $F^{t_{i}}=f^{s_{i}}$ on a neighbourhood of $c_{i}$. (In particular, $t_{i}=s_{i}=0$ for $1 \leq i \leq b^{\prime}$.)

For any $0 \leq h<\infty$, and any $c^{\prime} \in[c]$, let $a_{h}\left(c^{\prime}\right)$ be the minimal non-negative integer such that $f^{a_{h}\left(c^{\prime}\right)}\left(c^{\prime}\right) \in U_{1}^{h}$ and let $P_{h}\left(c^{\prime}\right)$ be the component of $f^{-a_{h}\left(c^{\prime}\right)}\left(U_{1}^{h}\right)$ containing $c^{\prime}$. Then $f^{a_{h}\left(c^{\prime}\right)}: P_{h}\left(c^{\prime}\right) \rightarrow U_{1}^{h}$ is a proper map of a uniformly bounded degree. Write $q_{h}=p_{1, h}+s_{k_{1, h}}$. Then for some $1 \leq i_{h} \leq b^{\prime}, f^{a_{h}\left(c^{\prime}\right)+q_{h}}: P_{h}\left(c^{\prime}\right) \rightarrow V_{i_{h}}$ is also a proper map of a uniformly bounded degree. For each $c^{\prime} \in[c]$, if $f^{a_{h}\left(c^{\prime}\right)+q_{h}}\left(c^{\prime}\right) \in U_{i_{h}}^{j_{h}}$, then let $Q_{h}\left(c^{\prime}\right)$ be the component of $f^{-\left(a_{h}\left(c^{\prime}\right)+q_{h}\right)}\left(U_{i_{h}}^{j_{h}}\right)$ which contains $c^{\prime}$.

LEMMA 5.1. For any $x \in J_{c}$ and any large $h$, if $n_{h}$ is the minimal non-negative integer such that $f^{n_{h}}(x) \in \bigcup_{d \in[c]} Q_{h}(d)$, and if $c^{\prime}=c_{h}^{\prime}$ is the critical point such that $f^{n_{h}}(x) \in Q_{h}\left(c_{h}^{\prime}\right)$, and if $A_{h}(x)$ is the component of $f^{-n_{h}}\left(P_{h}\left(c^{\prime}\right)\right)$ containing $x$, then the proper map $f^{n_{h}}: A_{h}(x) \rightarrow P_{h}\left(c^{\prime}\right)$ has a degree uniformly bounded from above.

Proof. Let $E_{k}^{h}=f^{k}\left(A_{h}(x)\right)$ for $0 \leq k \leq n_{h}$. We need to count the number of times a critical point appears in some $E_{k}^{h}$. Let $T_{k}^{h}=E_{k}^{h} \cap S^{1}$. Then $\left\{T_{k}^{h}\right\}_{k=0}^{n_{h}}$ is a (real) maximal chain. (We admit the situation that $T_{k}^{h}=\emptyset$ for some $k$.) Since all critical points of $f$ are contained in $S^{1}$, it suffices to show that the maximal chain $\left\{T_{k}^{h}\right\}_{k=0}^{n_{h}}$ has a uniformly bounded order. Note that for $h$ sufficiently large $\max _{k=0}^{n_{h}-1}\left|T_{k}^{h}\right|$ is small since $f \mid S^{1}$ has no wandering interval. So each $E_{k}^{h}$ contains, at most, one critical point. For the same reason, any $d \in C(f)-C_{r}(f)$ appears in, at most, one of the domains $E_{k}^{h}$.

Assume that the maximal chain has order larger than $2 \# C(f)$. Then there exists some $d \in C_{r}(f)$ such that $d \in T_{k_{1}}^{h}, T_{k_{2}}^{h}$ for some $0 \leq k_{1}<k_{2} \leq n_{h}$ and the maximal chain $\left\{T_{k}^{h}\right\}_{k=k_{1}}^{n_{h}}$ has order $\leq 2 \# C(f)$. Since $f^{n_{h}-k_{1}}: E_{k_{1}}^{h} \rightarrow P_{h}\left(c^{\prime}\right)$ has a degree bounded from above and $Q_{h}\left(c^{\prime}\right)$ has a small diameter in the hyperbolic Riemann surface $P_{h}\left(c^{\prime}\right)$, and since $\left|T_{k_{1}}^{h}\right|$ has a small diameter, $f^{k_{1}}(x)$ is close to $d$. If such a $d$ appears for infinitely many $h \mathrm{~s}$, then it is contained in $\omega(x)$. Since $x \in J_{c}$, we must have $d \in[c]$. So there are positive integers $m_{1}>m_{2}$ such that $f^{n_{h}-k_{1}}=F^{m_{1}}, f^{n_{h}-k_{2}}=F^{m_{2}}$ holds in a neighbourhood of $d$. It is then easy to see that $E_{k_{2}}^{h} \subset P_{h}(d)$ and $E_{k_{1}}^{h} \subset Q_{h}(d)$. Since $f^{k_{1}}(x) \in E_{k_{1}}^{h}$, this contradicts the minimality of $n_{h}$. Thus the maximal chain $\left\{T_{k}^{h}\right\}_{k=0}^{n_{h}}$ has order $\leq 2 \# C(f)$. The proof of the lemma is then completed.

We continue the proof of Proposition 5.4. By now, we have, for each $h$ large, a proper map $f^{n_{h}+a_{h}\left(c_{h}^{\prime}\right)+q_{h}}: A_{h}(x) \rightarrow V_{i_{h}}$ of a uniformly bounded degree such that $f^{n_{h}+a_{j}\left(c_{h}^{\prime}\right)+q_{h}}(x)$ is contained in $U_{i_{h}}^{j_{h}}$ for some $1 \leq i_{h} \leq b^{\prime}$ and $0 \leq j_{h}<r_{i_{h}}+1$. By the

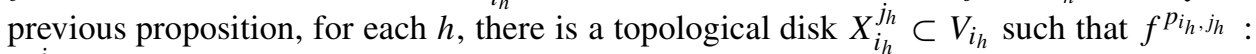
$X_{i_{h}}^{j_{h}} \rightarrow W_{k_{i_{h}, j_{h}}}$ is a proper map of a uniformly bounded degree. The proposition follows. $\square$ 
Proof of Theorem 5.1. Let $X \subset J_{c}$ be a measurable set of positive measure such that $f(X) \subset X(\bmod 0)$. Let $x \in X$ be a Lebesgue density point. Let $d \in[c]$, and $U \subset \subset U^{\prime}$ be neighbourhoods of $d$ as in the previous proposition. There exist $n_{1}<n_{2}<\cdots$ neighbourhoods $A_{j}$ of $x$, such that $f^{n_{j}}: A_{j} \rightarrow U^{\prime}$ has a uniformly bounded degree and $f^{n_{j}}(x) \in U$. Hence by Lemma 2.2 and $2.3, m(U-X)=0$. Since $f^{n}(U)=\hat{\mathbb{C}}$ $(\bmod 0)$ for some $n \in \mathbb{N}$, we have that $X=\hat{\mathbb{C}}(\bmod 0)$. Therefore $J(f)=\hat{\mathbb{C}}$ and $f$ is ergodic.

If $f$ carries an invariant line field $\mu$ on $J_{c}$, let $x$ be an almost continuous point of $\mu$. One can construct a sequence of proper mappings $f^{n_{j}}: A_{j} \rightarrow U^{\prime}$ as in the previous proof, which then implies that $\mu$ is holomorphic on a non-empty open set. Thus $f$ is doubly covered by an integral torus endomorphism. In particular, $f$ has no recurrent critical point. Contradiction!

Reduction. From now on, we shall assume that for any $c \in C_{r}(f)$ with $\omega(c)$ non-minimal, the set $J_{c}$ has measure zero. It follows from a similar argument as in the proof of Proposition 2.2 that for a.e. $z \in J(f), \omega(z) \subset \operatorname{Par}(f) \cup\left(\bigcup_{c \in C_{r}(f), \omega(c) \text { minimal }} \omega(c)\right)$. The last set is a disjoint union of finitely many minimal sets. Since the set $\{z \in J(f)$ : $\omega(z) \subset \operatorname{Par}(f)\}$ is countable, we may assume that:

(1) $\quad J(f)=\bigcup_{c \in C_{r}(f), \omega(c) \text { minimal }} J_{c}(\bmod 0)$; and

(2) $J_{c}=\{z \in J(f): \omega(z)=\omega(c)\}(\bmod 0)$ for any $c \in C_{r}(f)$ such that $\omega(c)$ is minimal.

6. Large real bound implies the non-existence of an invariant line field

We begin the study of the measurable dynamics of $f \mid J_{c}$ in the case that $\omega(c) \ni c$ is a minimal set. We shall assume the following throughout this section.

Assumption. There exists a sequence of symmetric nice intervals $I(n) \ni c, n=1,2, \ldots$, such that $|I(n)| \rightarrow 0$ and $d\left(\omega(c) \cap I(n), \omega(c)-I_{n}\right) / \operatorname{diam}(\omega(c) \cap I(n)) \rightarrow \infty$ as $n \rightarrow \infty$.

Let $b=\#[c]$, and $c_{1}=c, c_{2}, \ldots, c_{b}$ be the critical points contained in $[c]$.

PROPOSITION 6.1. There is a constant $\rho_{0}$ such that for any $\rho \geq \rho_{0}$ the following holds.

Let $I \ni c$ be a symmetric nice interval interval. Suppose that $|I|$ is very small and satisfies $d(\omega(c) \cap I, \omega(c)-I) \geq \rho \operatorname{diam}(\omega(c) \cap I)$, let $B_{I}:\left(\bigcup_{j=0}^{r} J^{j}\right) \cup\left(\bigcup_{i=2}^{b} I_{i}\right) \rightarrow$ $\bigcup_{i=1}^{b} I_{i}$ be the real box mapping associated to $I$. Then there is a quasi-polynomial-like mapping $\mathbf{F}=\left\{F: U_{i}^{j} \rightarrow \bigcup_{i=1}^{b} V_{i} \mid 1 \leq i \leq b, 0 \leq j<r_{i}+1\right\}$ corresponding to $c$ such that:

(i) $\quad r_{1}=r, r_{2}=r_{3}=\cdots=r_{b}=0$ and $U_{i}^{0}=V_{i}$ for any $2 \leq i \leq b$;

(ii) $V_{i} \cap \omega(c)=I_{i} \cap \omega(c), U_{1}^{j} \cap \omega(c)=J_{1}^{j} \cap \omega(c)$, for any $1 \leq i \leq b$ and any $0 \leq j \leq r$

(iii) $F\left|\left(V_{i} \cap I_{i}\right)=B_{I}\right|\left(V_{i} \cap I_{i}\right), F\left|\left(U^{j} \cap J^{j}\right)=B_{I}\right|\left(U^{j} \cap J^{j}\right)$, for any $2 \leq i \leq b$ and any $0 \leq j \leq r$;

(iv) there is topological disk $D$ containing $V_{1}$ such that $D \cap \omega(c)=V_{1} \cap \omega(c)$, and

$$
\bmod \left(D-\overline{V_{1}}\right) \geq m(\rho)>0,
$$

where $m(\rho) \rightarrow \infty$ as $\rho \rightarrow \infty$. 
Proof. We first assume that $r \neq 0$.

Since $d(\omega(c) \cap I, \omega(c)-I) / \operatorname{diam}(\omega(c) \cap I)$ is very large, there is a round annulus $X$ in $\hat{\mathbb{C}}-\omega(c)$ centred at $c$ separating $\omega(c) \cap I$ from $\omega(c)-I$ with a large modulus. Take the core curve $\gamma$ of $X$. It is a round circle centred at $c$. Let $E_{1}$ denote the topological disk bounded by $\gamma$. Let $k_{j}(0 \leq j \leq r), m_{i}(2 \leq i \leq b)$ be the positive integers such that $B_{I} \mid I_{i}=f^{m_{i}}$ and $B_{I} \mid J^{j}=f^{k_{j}}$. Let $m_{i}^{\prime}(2 \leq i \leq b)$ and $k_{j}^{\prime}(0 \leq j \leq r)$ be the first return time of $I_{i}, J^{j}$ to $I$, i.e. the minimal positive integers such that $f^{m_{i}^{\prime}}\left(I_{i}\right) \subset I$ and $f^{k_{j}^{\prime}}\left(J^{j}\right) \subset I$. Let $E_{i}$ be the component of $f^{-m_{i}^{\prime}}\left(E_{1}\right)$ containing $c_{i}, 2 \leq i \leq b$, where $c_{i}$ is the critical point of $f$ which is contained in $I_{i}$. For $0 \leq j \leq r$, let $D^{j}$ be the component of $f^{-k_{j}^{\prime}}\left(E_{1}\right)$ containing $\omega(c) \cap J^{j}$. It is easy to see that if $B_{I}\left(I_{i}\right) \subset I_{i^{\prime}}$, then $f^{m_{i}}\left(E_{i}\right)=E_{i^{\prime}}$ and that if $B_{I}\left(J^{j}\right) \subset I_{i^{\prime}}$, then $f^{k_{j}}\left(D^{j}\right)=E_{i^{\prime}}$.

We claim that $D^{j} \subset E_{1}$ for any $0 \leq j \leq r$.

Indeed, since $X-\overline{E_{1}}$ is an annulus with a large modulus $(=\bmod (X) / 2)$ which is disjoint from $\omega(c)$, it is easy to show by Koebe's distortion theorem that any $\Omega \in\left\{E_{i}, D^{j}: 1 \leq\right.$ $i \leq b, 0 \leq j \leq r\}$ is an 'almost round' disk as seen from any $x \in \Omega \cap \omega(c)$, that is

$$
\max _{z \in \partial \Omega} d(z, x) \leq(1+\epsilon) d(x, \partial \Omega)
$$

where $\epsilon>0$ can be taken close to 0 if $\rho$ is large. Since $r \geq 1$, we have $\operatorname{diam}\left(D^{j}\right) \leq$ $2(1+\epsilon) \operatorname{diam}(\omega(c) \cap I)$. Since $E_{1} \cap S^{1}$ is an interval containing a large neighbourhood of $\omega(c) \cap I$, it follows that $D^{j} \subset E_{1}$.

$\left\{F_{1, j}: U^{j} \rightarrow \bigcup_{i} V_{i}, F_{k}: V_{k} \rightarrow \bigcup_{i} V_{i} \mid 0 \leq j \leq r, 2 \leq k \leq b\right\}$ is a quasi-polynomiallike mapping satisfying the required conditions.

We now turn to the case that $r=0$.

In this case there is a permutation $\sigma:\{1,2, \ldots, b\} \rightarrow\{1,2, \ldots, b\}$ such that $B_{I}\left(I_{i}\right) \subset I_{\sigma(i)}$, where $B_{I}$ is the real box mapping associated to $I$, for otherwise the forward orbit of $c$ will not enter some of the intervals $I_{i}$, which contradicts the hypothesis that $\omega(c)$ contains all critical points in $\bigcup_{i=1}^{b} I_{i}$. By possibly changing the subscript $i$, we may assume that $\sigma(i)=i+1$ for any $1 \leq i \leq b-1$ and $\sigma(b)=1$. Let $k_{i}$ be the positive integer such that $B_{I} \mid I_{i}=f^{k_{i}}$. Let $m=k_{1}+k_{2}+\cdots+k_{b}$.

Construct $X, E_{1}$ as in the case $r \neq 0$. Let $D^{0}$ be the pre-image of $f^{-m}\left(E_{1}\right)$ containing $c$. $G=f^{m}: D^{0} \rightarrow E_{1}$ is a proper map. For any $u \in D_{0}$ such that $G^{\prime}(u)=0$, $G^{n}(u)$ never escapes the domain $D^{0}$. Write $P=\overline{\left\{G^{n}(u): G^{\prime}(u)=0, n \in \mathbb{N}\right\}}$, then $\bmod \left(P, E_{1}\right) \geq \bmod \left(\omega(c) \cap I, E_{1}\right) \geq \bmod (X) / 2$ is large. By Theorem 5.12 in [20], there is a domain $U^{\prime} \subset D^{0}$ such that $f^{m}: U^{\prime} \rightarrow V^{\prime}=f^{m}\left(U^{\prime}\right)$ is a polynomial-like map with the same degree as $G$ and $\bmod \left(V^{\prime}-\overline{U^{\prime}}\right)$ is large since $f$ has no attracting cycle in $D_{0}$. Define $U^{0}=U^{\prime}, V_{1}=V^{\prime}$ and $V_{s}=f^{k_{1}+k_{2}+\cdots+k_{s-1}}\left(U^{0}\right)$. Then $\left\{f^{k_{1}}: U^{0} \rightarrow V_{1}, f^{k_{i}}:\right.$ $\left.V_{i} \rightarrow V_{i+1}, f^{k_{b}}: V_{b} \rightarrow V_{1} \mid 2 \leq i \leq b-1\right\}$ is a quasi-polynomial-like mapping as required.

For $\delta>0$ let $\mathcal{E}^{\delta}$ be the collection of quasi-polynomial-like mappings $\left\{F_{i, j}: U_{i}^{j} \rightarrow\right.$ $\left.\bigcup_{i=1}^{b} V_{i} \mid 1 \leq i \leq b, 0 \leq j \leq r_{i}\right\}$ corresponding to $c$ induced by $f$ such that $r_{i}=0, U_{i}^{0}=V_{i}$ for all $2 \leq i \leq b$ and such that there is a topological disk $D$ containing $V_{1}$ such that $D \cap \omega(c)=V_{1} \cap \omega(c)$ and $\bmod \left(D-\overline{V_{1}}\right) \geq \delta$. 
For each quasi-polynomial-like mapping $\mathbf{F}$ in $\mathcal{E}^{\delta}$, and each $2 \leq i_{0} \leq b$, we can associate to it another quasi-polynomial-like mapping $\tilde{\mathbf{F}}$ in the following way. Let $c_{1} \in U^{0}, c_{i} \in V_{i}$ be the critical points of $f$. Denote $\tilde{V}_{1}=V_{i_{0}}$. For each $1 \leq i^{\prime} \leq b, i^{\prime} \neq i_{0}$, let $k\left(i^{\prime}\right)$ be the minimal positive integer such that $F^{k\left(i^{\prime}\right)}\left(c_{i^{\prime}}\right) \in \tilde{V}_{1}$ and let $\tilde{V}_{i^{\prime}}=\operatorname{Comp}_{c_{i^{\prime}}}\left(\mathbf{F}, k\left(i^{\prime}\right), \tilde{V}_{1}\right)$. For each $x \in \omega(c) \cap \tilde{V}_{1}$, let $k(x)$ be the minimal positive integer such that $F^{k(x)}(x) \in \tilde{V}_{i}$ for some $1 \leq i \leq b$, and let $\tilde{U}(x)=\operatorname{Comp}_{x}\left(\mathbf{F}, k(x), \tilde{V}_{i}\right)$. Then define

$\left.\tilde{\mathbf{F}}=\left\{F^{k(x)}: \tilde{U}(x)\right) \rightarrow \bigcup_{i=1}^{b} \tilde{V}_{i}, F^{k\left(i^{\prime}\right)}: \tilde{V}_{i^{\prime}} \rightarrow \bigcup_{i=1}^{b} \tilde{V}_{i} \mid x \in \omega(c) \cap \tilde{V}_{1}, 1 \leq i^{\prime} \leq b, i^{\prime} \neq i\right\}$.

Note that there is a constant $\tilde{\delta}>0$ depending only on $\delta$ (and $f$ ) such that $\tilde{F} \in \mathcal{E}^{\delta}$. Moreover, we can take $\tilde{\delta}$ arbitrarily large if $\delta$ is sufficiently large.

THEOREM 6.2. If there is a sequence of symmetric nice intervals $I_{n} \ni c$ such that

(1) $\left|I_{n}\right| \rightarrow 0$ as $n \rightarrow \infty$; and

(2) $\quad d\left(\omega(c) \cap I_{n}, \omega(c)-I_{n}\right) / \operatorname{diam}\left(\omega(c) \cap I_{n}\right) \rightarrow \infty$ as $n \rightarrow \infty$, then

(i) if $m\left(J_{c}\right)>0$, then $f \mid J_{c}$ is ergodic; and

(ii) $J_{c}$ carries no $f$-invariant line field.

Proof. By Proposition 6.1, there is a sequence of quasi-polynomial-like mappings

$$
\begin{aligned}
F(n)=\left\{F(n)_{1, j}: U^{j}(n) \rightarrow \bigcup_{i=1}^{b} V_{i}(n) ;\right. & F(n)_{k, 0}: V_{k}(n) \\
& \left.\rightarrow \bigcup_{i=1}^{b} V_{i}(n) \mid 0 \leq j \leq r(n), 1 \leq k \leq b\right\}
\end{aligned}
$$

corresponding to $c$ (induced by $f$ ) which are contained in the class $\mathcal{E}^{\delta_{n}}$ for $\delta_{n} \rightarrow \infty$ as $n \rightarrow \infty$ such that $\operatorname{diam}\left(V_{1}(n)\right) \rightarrow 0$ as $n \rightarrow \infty$. Let $W(n) \supset V_{1}(n)$ be a topological disk such that $W(n) \cap \omega(c)=V_{1}(n) \cap \omega(c)$ and $\bmod \left(W(n)-\overline{V_{1}(n)}\right) \geq \delta_{n}$.

(i) Let $E \subset J_{c}$ be a measurable set of positive measure with $f(E) \subset E(\bmod 0)$ and let $x$ be a Lebesgue density point of $E$ such that $\omega(x)=\omega(c)$ and $f^{n}(x) \notin C(f)$ for any $n \geq 0$.

For any $n \in \mathbb{N}$, let $k(n)$ be the minimal non-negative integer such that $f^{k(n)}(x) \in$ $U^{0}(n) \cup\left(\bigcup_{i=2}^{n} V_{i}(n)\right)$ and let $1 \leq i(n) \leq b$ be such that $f^{k(n)} \in V_{i(n)}(n)$. Then $k(n) \rightarrow \infty$ as $n \rightarrow \infty$ since $\max _{i=1}^{b} \operatorname{diam}\left(V_{i}(n)\right) \rightarrow 0$. Let $A(n)$ be the component of $f^{-k(n)}\left(V_{i(n)}(n)\right)$ containing $x$. Let $p(n)$ be the positive integer such that $f^{p(n)}\left(V_{i(n)}(n)\right)=V_{1}(n)$ if $i(n) \neq 1$ and let $p(n)=0$ otherwise. Let $X(n)$ be the component of $f^{-p(n)}(W(n))$ containing $V_{i(n)}(n)$ and let $A^{\prime}(n)$ be the component of $f^{-k(n)}(X(n))$ containing $A(n)$. Then $f^{p(n)}: X(n) \rightarrow W(n)$ is a proper map of a uniformly bounded degree, and $X(n) \cap \omega(c)=V_{i(n)}(n) \cap \omega(c)$. We claim that for $n$ sufficiently large, $f^{k_{n}}: A^{\prime}(n) \rightarrow X(n)$ is also a conformal map.

Assume that the claim fails. Let $k<k(n)$ be the maximal number such that $f^{k}\left(A^{\prime}(n)\right)$ contains a critical point $d$ of $f$. First let us show that $d \in \omega(c)$. Since $f(d) \in f^{k+1}\left(A^{\prime}(n)\right)$, $f^{k+1}\left(A^{\prime}(n)\right) \cap S^{1} \neq \emptyset$. Since $f^{k(n)-k-1} \mid f^{k+1}\left(A^{\prime}(n)\right)$ is a conformal map, $f^{k(n)-k-1}$ : $f^{k+1}\left(A^{\prime}(n)\right) \cap S^{1} \rightarrow X(n) \cap S^{1}$ is a diffeomorphism. In particular, $f^{k+1}(A(n)) \cap S^{1} \neq \emptyset$. 
Since $\operatorname{diam}\left(f^{k+1}(A(n))\right)$ and $\left|f^{k+1}\left(A^{\prime}(n)\right) \cap S^{1}\right|$ are small, we have that $f^{k+1}(x)$ is close to $f(d)$, and hence $f^{k}(x)$ is close to $d$. So we have $d \in \omega(x)=\omega(c)$ if $n$ is large. Since $X(n) \cap \omega(c)=V_{i(n)}(n) \cap \omega(c), d \in f^{k}(A(n))$. Since $f^{k(n)-k}(d) \in \bigcup_{i=1}^{b} V_{i}(n)$, there is some positive integer $t$ such that $F(n)^{t}$ is well defined in a neighbourhood of $d$ and coincides with $f^{k(n)-k}$. Then it is easy to see that $f^{k}(A(n)) \subset U^{0}(n) \cup \bigcup_{i=2}^{b} V_{i}(n)$. Since $f^{k}(x) \in f^{k}(A(n))$, this contradicts the minimality of $k(n)$.

Thus $f^{k(n)+p(n)}: A^{\prime}(n) \rightarrow W(n)$ is a proper map of a uniformly bounded degree. Since $x$ is a Lebesgue density point and $f^{k(n)+p(n)}(x) \in V(n)$, it follows that $m(E \cap$ $V(n)) / m(V(n)) \rightarrow 1$ as $n \rightarrow \infty$.

If $m\left(J_{c}\right)>0$ and $f \mid J_{c}$ is not ergodic, then there is a measurable set $E \subset J_{c}$ such that $f^{-1}(E)=E(\bmod 0)$ and $m(E)>0, m\left(J_{c}-E\right)>0$. Then it follows that $m(E \cap V(n)) / m\left(V_{n}\right) \rightarrow 1$ and $m\left(\left(J_{c}-E\right) \cap V(n)\right) / m(V(n)) \rightarrow 1$ as $n \rightarrow \infty$, which is obviously absurd.

(ii) Assume that $f$ carries an invariant line field $\mu$ with support $E \subset J_{c}$. We need to show a contradiction.

Let $x \in E$ be an almost continuous point of $\mu$ such that $\mu(x) \neq 0$ and $f^{n}(x) \notin C(f)$ for any $n \geq 0$. By the same argument as in the previous proof, for each $n$ large, there are $k(n) \in \mathbb{N}, 1 \leq i(n) \leq b$ such that $f^{k(n)}(x) \in V_{i(n)}(n)$ and there is a univalent branch $h(n)$ of $f^{-k(n)}$, defined on $V_{i(n)}(n)$, mapping $f^{k(n)}(x)$ to $x$.

Case 1 . There are infinitely many $n$ such that $i(n)=1$.

By passing to a subsequence, we may assume that $i(n)=1$ for all $n \in \mathbb{N}$. Let $s(n)$ be the first return time of $U^{0}(n)$ to $V_{1}(n)$. Then $f^{s_{n}}: U^{0}(n) \rightarrow V_{1}(n)$ is a proper map whose degree is no less than 2 and bounded uniformly from above.

Since $f^{k(n)}(x) \in U^{0}(n) \subset V_{1}(n)$, it follows from Corollary 3.3 that this is impossible.

Case 2. For $n \gg 1, i(n)>1$.

Let

$$
\tilde{F}(n):\left(\bigcup_{j=0}^{\tilde{r}(n)} \tilde{U}^{j}(n)\right) \cup\left(\bigcup_{i=2}^{b} \tilde{V}_{i}(n)\right) \rightarrow \bigcup_{i=1}^{b} \tilde{V}_{i}(n)
$$

be the holomorphic box mapping associated to $F(n)$ and $i(n)$ as in the remark before Corollary 7.3. Then $\tilde{F}(n)$ belongs to the class $\mathcal{E}^{\tilde{\delta}_{n}}$ with $\tilde{\delta}_{n} \rightarrow \infty$. Let $\tilde{k}(n)$ be the minimal non-negative integer such that

$$
f^{\tilde{k}(n)}(x) \in \tilde{U}^{0}(n) \cup\left(\bigcup_{i=2}^{b} \tilde{V}_{i}(n)\right)
$$

and let $1 \leq \tilde{i}(n) \leq b$ be such that $f^{\tilde{k}(n)}(x) \in \tilde{V}_{\tilde{i}(n)}(n)$.

If there are infinitely many $n$ such that $\tilde{i}(n)=1$, then we return to case 1 and the proof is completed. So let us assume that for $n \gg 1, \tilde{i}(n) \neq 1$. So there is a univalent branch of $\tilde{h}(n): \tilde{V}_{\tilde{i}(n)}(n) \rightarrow \hat{\mathbb{C}}$ of $f^{-\tilde{k}(n)}$ such that $\tilde{h}(n)\left(f^{\tilde{k}(n)}(x)\right)=x$. Let $\tilde{s}(n)$ be the first return time of $\tilde{V}_{\tilde{i}(n)}(n)$ to $\tilde{V}_{1}(n)$. Then $f^{\tilde{s}(n)}: \tilde{V}_{\tilde{i}(n)}(n) \rightarrow \tilde{V}_{1}(n)$ is a proper map whose degree is no less than 2 and bounded from above uniformly. A contradiction again follows from Corollary 3.3. 
7. Bounded geometry implies the non-existence of an invariant line field on the Julia set We fix an $f \in \mathcal{F}$ and $c \in C_{r}(f)$ with $\omega(c)$ minimal. Assume that there is a constant $M>0$ such that for any symmetric nice interval $I$ containing $c$, we have

$$
\frac{d(\omega(c) \cap I, \omega(c)-I)}{\operatorname{diam}(\omega(c) \cap I)} \leq M
$$

By taking $M$ larger, we may assume that the inequality (6) holds for any small nice interval intersecting $\omega(c)$.

To conclude the proof of Theorem 1 and 2, it suffices to prove the following theorem.

THEOREM 7.1. In the previous situation, we have:

(1) $m\left(J_{c}\right)=0$ or $f \mid J_{c}$ is ergodic;

(2) $f$ carries no invariant line field on $J_{c}$.

Since $f$ has a recurrent critical point, it has infinitely many periodic points on the extended real axis $S^{1}$. Using conjugacy by real Möbius transformation, we may assume that $\infty$ is a periodic point of $f$.

Take an arbitrarily small symmetric interval $T \ni c$ and let $B_{T}:\left(\bigcup_{j=0}^{r} S^{j}\right) \cup$ $\left(\bigcup_{i=2}^{b} T_{i}\right) \rightarrow \bigcup_{i=1}^{b} T_{i}$ be the real box mapping associated to $T$. As described in $\S 4$, this real box mapping can be extended to a quasi-polynomial-like mapping $\mathbf{F}=\left\{F_{1, j}: U_{i}^{j} \rightarrow\right.$ $\left.\bigcup_{k=1}^{b} V_{k} \mid 1 \leq i \leq b, 0 \leq j \leq r_{i}\right\}$ with $V_{i}=\hat{\mathbb{C}}_{T_{i}}$ and $r_{1}=r, r_{2}=r_{3}=\cdots=r_{b}=0$. We shall fix $\mathbf{F}$ from now on. Let $F$ be the map determined by $\mathbf{F}$ as described at the top of page 967.

From now on, all metrics are the Euclidean metric in $\mathbb{C}$ unless otherwise stated. All intervals are assumed to be contained in $\bigcup_{i=1}^{b} T_{i}$. For any two disjoint open intervals $A_{1}, A_{2}$, we shall use $\left(A_{1}, A_{2}\right)$ to denote the maximal bounded open interval which is disjoint from $A_{1} \cup A_{2}$, and similarly use $\left(A_{1}, A_{2}\right.$ ] to denote the minimal open interval which contains $\left(A_{1}, A_{2}\right) \cup A_{2}$.

For any $\delta>0$, let $\mathcal{I}_{\delta}$ be the collection of intervals $I$ satisfying the following conditions: $I$ is a symmetric nice interval $I$ containing a critical point in $\omega(c)$ such that the $\delta$-neighbourhood of $I$ is disjoint from $\omega(c)-I$; moreover, if $f$ is only finitely renormalizable at $c, I$ is a $\delta$-nice interval and if $f$ is infinitely renormalizable at $c, I$ is a properly periodic interval. By Proposition 3.1 and Corollary 4.7 in [26], for some $\delta_{0}>0$, $\mathcal{I}_{\delta_{0}}$ contains an arbitrarily small interval containing $c$. (By saying that $I$ is a $\delta$-nice interval, we mean that for any $x \in \omega(c) \cap I$, if $J$ is the component of the domain of the first return map to $I$, then $I$ contains the $\delta$-neighbourhood of $J$.)

Let $I$ be a small interval in $\mathcal{I}_{\delta_{0}}$ and let $J_{i}, i=1, \ldots, n$ be the components of the domain of the first return map to $I$ which (are contained in $\bigcup_{k} T_{k}$ and) intersect $\omega(c)-I$ and let $J_{0}=I$.

LEMMA 7.1. There is a constant $\delta_{1}>0$ such that for any $0 \leq i \leq n$,

$$
\frac{d\left(J_{i}, \omega(c)-J_{i}\right)}{\left|J_{i}\right|} \geq \delta_{1} .
$$

Proof. For any $i \neq 0$, let $r=r_{i}$ be the minimal positive integer such that $f^{r}\left(J_{i}\right) \subset I$. Let $I^{\prime}$ denote the $\delta_{0}$-neighbourhood of $I$ and let $D=D\left(I^{\prime}\right)$. Let $U$ be the component of 
$f^{-r}(D)$ which contains $J_{i}$. Then $f^{k}: U \rightarrow D$ is a proper map with uniformly bounded degree. Obviously, $U \cap \mathbb{R}$ contains a definite neighbourhood of $J_{i}$, and is disjoint from $\omega(c)-J_{i}$.

For any $0 \leq i \leq n$, let $1 \leq k_{i} \leq b$ be such that $J_{i} \subset T_{k_{i}}$.

LEMMA 7.2. There exists $0 \leq i, j \leq n$ with $i \neq j, k_{i}=k_{j}$, such that there is no point in $\omega(c)$ between $J_{i}$ and $J_{j}$, and such that

$$
d\left(J_{i}, J_{j}\right) \leq C_{1} \min \left(\left|J_{i}\right|,\left|J_{j}\right|\right), \quad C_{1}^{-1}\left|J_{j}\right|<\left|J_{i}\right|<C_{1}\left|J_{j}\right|,
$$

where $C_{1}$ is a constant depending only on $\delta_{1}$ and $f$.

Proof. Take $0 \leq i \leq n$ be such that

$$
\left|J_{i}\right|=\min _{m=0}^{n}\left|J_{m}\right|
$$

First assume that both components of $T_{k_{i}}-J_{i}$ contain an interval of the form $J_{j}$. Let $j_{1} \neq j_{2}$ be such that $J_{j_{1}}, J_{j_{2}} \subset T_{k_{i}}$ and such that $\left(J_{i}, J_{j_{1}}\right)$ and $\left(J_{i}, J_{j_{2}}\right)$ are disjoint from $\bigcup_{m} J_{m}$. It cannot happen that both $\left(J_{i}, J_{j_{1}}\right]$ and $\left(J_{i}, J_{j_{2}}\right]$ are much larger than $\mid J_{i}$, for otherwise, a large neighbourhood of $J_{i}$ is disjoint from $\omega(c)-J_{i}$, which contradicts assumption (6). Thus the lemma holds. Now let us consider the case that only a component, say $L$, of $T_{k_{i}}-J_{i}$ intersects $\bigcup_{m} J_{m}$. Let $j$ be such that $J_{j} \subset L$ is closest to $J_{i}$. When $I$ is small, $J_{i}$ is also small, and hence the other component of $T_{k_{i}}-J_{i}$ is much larger than $J_{i}$. Similarly as before, we must have $\left(J_{i}, J_{j}\right]$ is not too large compared to $J_{i}$. So the lemma holds in this case as well.

We shall say that a topological disk $P$ is admissible if $\partial P$ is a smooth curve disjoint from $\omega(c)$, and if one of the following holds:

(A1) $P \cap \omega(c)=\emptyset$; or

(A2) there exists a nice interval $A \subset P$ such that $P \cap \omega(c)=A \cap \omega(c)$.

Let $C>1$ be a constant and let $P$ be a admissible topological disk. We say that $P$ is $C$-bounded if the following hold:

(D1) $l(\partial P)^{2} \leq C$ area $(P)$;

(D2) for $x \in \omega(c) \cap P_{i}$, and each $z \in P_{i}$, we have

$$
d(x, z) \leq C d\left(x, \partial P_{i}\right)
$$

(D3) $\bmod (\omega(c) \cap P, P) \geq C^{-1}$;

(D4) there is a topological disk $Q \supset \bar{P}$, such that $(Q-P) \cap \omega(c)=\emptyset$, and

$$
\bmod (Q-\bar{P}) \geq C^{-1} \text {. }
$$

We shall often consider a couple $\left(P_{1}, P_{2}\right)$ of admissible topological disks. We shall say that the couple $\left(P_{1}, P_{2}\right)$ is $C$-bounded if the following hold:

(T1) $P_{1} \cap P_{2} \cap \omega(c)=\emptyset$;

(T2) both of $P_{1}$ and $P_{2}$ are $C$-bounded;

(T3) $C^{-1} \operatorname{diam}\left(P_{2}\right) \leq \operatorname{diam}\left(P_{1}\right) \leq C \operatorname{diam}\left(P_{2}\right)$; and 
(T4) there is a piecewise smooth curve $\gamma$ which joins $P_{1}$ and $P_{2}$, such that

$$
l(\gamma) \leq C \operatorname{diam}\left(P_{1}\right)
$$

and for each $p \in \gamma$, we have

$$
B\left(p, C^{-1} \operatorname{diam}\left(P_{1}\right)\right) \cap \omega(c)=\emptyset .
$$

For instance, for any $0 \leq i \leq n$, the Poincaré disk $D\left(\left(1+\delta_{1} / 2\right) J_{i}\right)$ is a $C$-bounded admissible disk for some $C>1$. For $i, j$ as in Lemma $7.2\left(D\left(\left(1+\delta_{1} / 2\right) J_{i}\right), D((1+\right.$ $\left.\left.\delta_{1} / 2\right) J_{j}\right)$ ) is $C$-bounded for some $C>1$.

LEMMA 7.3. Let $P$ be a C-bounded admissible topological disk, and let $p$ be a positive integer. Let $P^{\prime}$ be a pull-back of $P$ under $\mathbf{F}$ and assume that the corresponding map $F^{n}: P^{\prime} \rightarrow P$ has degree at most $p$. Then $P^{\prime}$ is a $C^{\prime}$-bounded admissible topological disk, where $C^{\prime}=C^{\prime}(C, p)$ is a constant.

Proof. First of all, it is easy to check that $P^{\prime}$ is admissible.

Let $Q$ be a topological disk with $Q \cap \omega(c)=P \cap \omega(c)$ such that $\bmod (Q-P) \geq 1 / C$. Let $Q^{\prime} \supset P^{\prime}$ be the corresponding pull-back of $Q$. Then $F^{n}: Q^{\prime} \rightarrow Q$ obviously has the same degree as $F^{n} \mid P^{\prime}$. The lemma follows easily.

Now let $i, j$ be as in Lemma 7.2. Let $p_{i}$ be minimal non-negative integers such that $F^{p_{i}}\left(J_{i}\right) \subset I$, and let $p_{j}$ be similarly defined. Let

$$
\begin{aligned}
& E_{1}=\operatorname{Comp}_{J_{i}}\left(p_{i}, D\left(\left(1+\delta_{1} / 2\right) I\right)\right), \\
& E_{2}=\operatorname{Comp}_{J_{j}}\left(p_{j}, D\left(\left(1+\delta_{1} / 2\right) I\right)\right) .
\end{aligned}
$$

We can easily show that $\left(E_{1}, E_{2}\right)$ is a $C_{2}$-bounded couple of admissible topological disks. Indeed, we can take the interval $\left(J_{i}, J_{j}\right)$ to be the curve joining $E_{1}$ and $E_{2}$. Moreover, $E_{1} \cup E_{2}$ is contained in a $C_{3}$-bounded topological disk $D$ with $D \cap \omega(c) \subset E_{1} \cup E_{2}$.

Let $k$ be the minimal non-negative integer such that $F^{k}(d) \in E_{1} \cup E_{2}$ for some critical point $d \in \omega(c)$. To fix the notation, we assume that $F^{k}(d) \in E_{1}$.

Let $D^{\prime}=\operatorname{Comp}_{d}(k, D)$, and $\Omega_{1}=\operatorname{Comp}_{d}\left(k, E_{1}\right)$. Then $\Omega_{1}$ is a $C_{3}$-bounded topological disk by Lemma 7.3. Write $\left(a_{k}, b_{k}\right)=\left(J_{i}, J_{j}\right)$ such that $a_{k} \in E_{1}$. Let $a \in \Omega_{1}$ be a point such that $F^{k}(a)=a_{k}$. Let $\gamma^{\prime} \subset D^{\prime}$ be an arbitrary lift with initial point $a$ of the curve $\gamma=\left(a_{k}, b_{k}\right)$ under $F^{k}$, and let $b$ be the endpoint of $\gamma^{\prime}$. Let $\Omega_{2}$ be the topological disk in $D^{\prime}$ such that $b \in \Omega_{2}$ and such that $F^{k}\left(\Omega_{2}\right)=E_{2}$.

Let $K$ be the real pull-back of $J_{i}$ along the orbit $\left\{d, F(d), \ldots, F^{k}(d)\right\}$. This is a symmetric nice interval contained in $\Omega_{1}$. Let $0 \leq p_{j}^{\prime} \leq p_{j}$ be minimal such that $L=F^{p_{j}^{\prime}}\left(J_{j}\right)$ contains a critical point. Note that $L$ is also a symmetric nice interval and is of the form $J_{j^{\prime}}$ for some $0 \leq j^{\prime} \leq n$. Let $\Omega_{2}^{\prime}=F^{p_{j}^{\prime}+k}\left(\Omega_{2}\right)=F^{p_{j}^{\prime}}\left(E_{2}\right)$. Note that for some $\delta_{2}>0, K, L \in \mathcal{I}_{\delta_{2}}$.

Note that $F^{k} \mid D^{\prime}$ has a bounded distortion outside a small neighbourhood of $\omega(c) \cap \Omega_{1}$.

LEMMA 7.4. The couple $\left(\Omega_{1}, \Omega_{2}\right)$ of admissible topological disks is $C_{4}$-boundedfor some $C_{4}>1$. 
Let $K$ and $L$ be the symmetric nice intervals constructed as before. In the case that $f$ is only finitely renormalizable at $c$, since both of $K$ and $L$ are in $\mathcal{I}_{\delta_{2}}$, by Theorem 1.4 in [26] and by assumption (6), each of $K$ and $L$ has, at most, $N=N(\delta)$ children. (If $\left\{G_{i}\right\}_{i=0}^{p}$ is a unimodal chain such that both $G_{p}$ and $G_{0}$ are symmetric nice intervals containing a critical point in $\omega(c)$, then we say that $G_{0}$ is a child of $G_{p}$.) This holds in the case that $f$ is infinitely renormalizable at $c$ as well: in fact, $K, L, I$ are then all periodic intervals and hence each of them has only one child.

Let

$$
B_{K}:\left(\bigcup_{j=0}^{r} K_{1}^{j}\right) \cup\left(\bigcup_{i=2}^{b} K_{i}\right) \rightarrow \bigcup_{i=1}^{b} K_{i}
$$

denote the real box mapping associated to $K$. And let

$$
R=R_{K}:\left(\bigcup_{j=0}^{r} K_{1}^{j}\right) \cup\left(\bigcup_{i=2}^{b} K_{i}\right) \rightarrow K_{1}=K
$$

denote the first return map to $K$. Write $R \mid K_{1}^{j}=f^{s_{1}^{j}}$ and denote by $D_{j}$ the components of $f^{-s_{1}^{j}}\left(\Omega_{1}\right)$ containing $K_{1}^{j}$. For $2 \leq i \leq b$, write $R\left|K_{i}=f^{s_{i}}\right| K_{i}$ and let $\tilde{\Omega}_{i}$ be the component of $f^{-s_{i}}\left(\Omega_{1}\right)$ containing $K_{i}$. Let $s_{i}^{\prime} \leq s_{i}$ be the positive integer such that $B_{K} \mid K_{i}=f^{s_{i}^{\prime}}$.

Proposition 7.2. Let $x \in J_{c} \cap K(\mathbf{F})$ be a point such that $F^{m}(x) \notin \omega(c)$ for any $m \geq 0$. Assume that $I$ is sufficiently small. Then there is a constant $C_{5}>1$ depending only on $f$, a domain $\Omega \in\left\{\tilde{\Omega}_{i}, 2 \leq i \leq b ; \Omega_{1} ; \Omega_{2}\right\}$ and a non-negative integer $k$ such that there is $a$ univalent branch $h$ of $f^{-k}$ defined on $\Omega$ and

$$
d(x, h(\Omega)) \leq C_{5} \operatorname{diam}(h(\Omega)) .
$$

Since the proof of this proposition is relatively long, we first show how it implies Theorem 7.1.

Proof of Theorem 7.1. (1) Assume that $X_{i} \subset J_{c}$ are two measurable sets of positive measure such that $f\left(X_{i}\right) \subset X_{i}(\bmod 0), i=1,2$. Assume that $m\left(X_{1} \cap X_{2}\right)=0$, and we shall deduce a contradiction.

Let $I \in \mathcal{I}_{\delta_{0}}$ be a small interval as before. Let $y \in X_{1}$ be a Lebesgue density point of $X_{1}$ such that $f^{m}(y) \notin \omega(c)$ for all $m \geq 0$. We also assume that $f^{m}(y)$ are all Lebesgue density points of $X_{1}$. Then it is not difficult to show that $x=f^{m}(y) \in K(\mathbf{F})$ for some positive integer $m$. Let $\Omega$ and $h$ be as desired by Proposition 7.2 for $I$ and $x$. Note that there is a positive integer $p$, such that $f^{p}: h(\Omega) \rightarrow D=D\left(\left(1+\delta_{1} / 2\right) I\right)$ is a proper map of uniformly bounded degree. When $I$ is sufficiently small, we have that $m\left(h(\Omega) \cap X_{1}\right) / m(h(\Omega))$ is close to 1 and hence so is $m\left(D \cap X_{1}\right) / m(D)$. Similarly, we can prove that $m\left(D \cap X_{2}\right) / m(D)$ is close to 1 , which is obviously absurd.

(2) If $f$ carries an invariant line field $\mu$ on $J_{c}$, then there is a point $x \in K(\mathbf{F})$ such that $f^{m}(x) \notin \omega(c)$ for all $m$, and such that $\mu(x) \neq 0$, and $\mu$ is almost continuous at $x$. Given any small interval $I \in \mathcal{I}_{\delta_{0}}$ as before, again let $\Omega$ be the domain as desired by Proposition 7.2 for $I$ and $x$. 
Note that there is a non-negative integer $p$, and a symmetric interval $A \in \mathcal{I}_{\delta^{\prime}}$ for some $\delta^{\prime}>0$ which is uniformly bounded from zero, such that $f^{p} \mid \Omega$ is a conformal map onto a $C$-bounded admissible topological disk $Q$ with $A \subset Q$ and $Q \cap \omega(c) \subset A$. Indeed, if $\Omega \neq \Omega_{2}$, then we just take $p=0$. In $\Omega=\Omega_{2}$, then by the previous construction, the statement is still true with $A=L$.

Thus, there is a univalent branch $g$ of $f^{k+p}$, defined on $Q$, such that $d(x, Q) / \operatorname{diam}(Q)$ is uniformly bounded. Obviously, such a map $g$ extends to a univalent map defined on $D=\mathbb{C}_{A^{\prime}}$, where $A^{\prime}$ is the maximal open interval with $A^{\prime} \cap \omega(c)=A \cap \omega(c)$.

Let $e$ be the critical point in $A$, and let $A_{0} \ni e$ be the component of the domain of the first return map to $A$. Then by Proposition 6.3 in [26], we have that $\left|A_{0}\right| /|A|$ is uniformly bounded from zero. Let $s$ be the return time of $A_{0}$ to $A$. Let $Y=D\left(\left(1+\delta^{\prime} / 2\right) A\right)$ and let $X$ be the component of $f^{-s}(Y)$ which contains $A_{0}$. Then $f^{s}: X \rightarrow Y$ is a proper map which has a critical point and is of uniformly bounded degree. Note that $\bmod (D-X \cup Y)$ is uniformly bounded from zero, and hence $g$ has a uniformly bounded distortion on $X \cup Y$.

Let $U=g(X)$ and $V=g(Y)$, and let $h=g \circ f^{s} \circ g^{-1}: U \rightarrow V$. Then $h \in \mathcal{H}(f)$ is a proper map with a critical point and of bounded degree. Since $\max _{z \in Y} d(e, z) / d(e, \partial Y)$ is uniformly bounded, so is $\max _{z \in U} d(g(e), z) / d(g(e), \partial U)$. Similarly, we have a uniform upper bound on $\max _{z \in V} d\left(z, g\left(f^{s}(e)\right)\right) / d\left(g\left(f^{s}(e)\right), \partial V\right)$. Also, since $d(U, x), d(V, x)$ are not so large compared to $\operatorname{diam}(g(Q))$, and $\operatorname{since} \operatorname{diam}(Q) \asymp \operatorname{diam}(Y) \asymp \operatorname{diam}(X)$, we have a uniform bound on $d(U, x) / \operatorname{diam}(U)$ and on $d(x, V) / \operatorname{diam}(V)$.

Choose a sequence of intervals $I_{m} \in \mathcal{I}_{\delta_{0}}$ such that $\left|I_{m}\right| \rightarrow 0$ as $m \rightarrow \infty$. Define $D_{m}$, $X_{m}, Y_{m}, s_{m}, g_{m}, h_{m}$ as before, and apply Proposition 3.2, we obtain a contradiction.

Proof of Proposition 7.2. The proof will be divided in two cases. Case 1 will be proved similarly to the case with decay geometry (a large real bound), while case 2 has to be proved differently.

LEMMA 7.5. For any $x \in \bigcup_{j=0}^{r} D_{j}-\Omega_{1}$, there is a piecewise smooth curve $\gamma$ joining $x$ to $\partial \Omega_{1}$, such that

(1) $l(\gamma) \leq \delta_{3}^{-1} \operatorname{diam}\left(\Omega_{1}\right)$;

(2) for any $z \in \gamma$, we have

$$
B\left(z, \delta_{3} \operatorname{diam}\left(\Omega_{1}\right)\right) \cap \omega(c)=\emptyset,
$$

where $\delta_{3}>0$ is a constant.

Proof. Let $K^{\prime}$ be the maximal open symmetric interval such that $K^{\prime} \cap \omega(c)=K \cap \omega(c)$. By (6), we know that the diameter of $K^{\prime}$ is comparable to the diameter of $\Omega_{1}$. Let $A=\hat{\mathbb{C}}_{K^{\prime}}$. For any $0 \leq j \leq r$, let $A_{j}$ be the component of $f^{-s_{1}^{j}}(A)$ which contains $K_{1}^{j}$. Then $f^{s_{1}^{j}}$ : $A_{j} \rightarrow A$ has the same degree as $f^{s_{1}^{j}}: D_{j} \rightarrow \Omega_{1}$ and hence is uniformly bounded from above. Since the hyperbolic diameter of $\Omega_{1}$ in $A$ is uniformly bounded, so is the hyperbolic diameter of $D_{j}$ in $A_{j}$, by Lemma 2.1 .

We claim that $A_{j} \subset A$ for any $0 \leq j \leq r$. Let $\tau: K \rightarrow K$ be the involution such that $f \circ \tau=f$. Consider the chain $\left\{G_{m}^{\prime}\right\}_{m=0}^{s_{1}^{j}}$ and also the chain $\left\{G_{m}\right\}_{m=0}^{s_{1}^{j}}$ with $G_{s_{1}^{j}}^{\prime}=K^{\prime}, G_{s_{1}^{j}}=K$ and $G_{0}^{\prime} \supset G_{0} \supset K_{1}^{j}$. Since $K^{\prime}-K$ is disjoint from $\omega(c)$, so is 
$G_{1}^{\prime}-G_{1}$. Thus both $G_{0}^{\prime}-G_{0}$ and $\tau\left(G_{0}^{\prime}\right)-\tau\left(G_{0}\right)$ are disjoint from $\omega(c)$. So $G_{0}^{\prime} \subset K^{\prime}$. Since $A_{j} \cap \mathbb{R}=G_{0}^{\prime}$, we have $A_{j} \subset \hat{\mathbb{C}}_{G_{0}^{\prime}} \subset A$.

Thus, the hyperbolic diameter of $D_{j}$ in $A$ is uniformly bounded from above. The lemma follows easily.

\section{Continuation of proof of Proposition 7.2.}

Case 1. For any non-negative integer $k$, we have

$$
F^{k}(x) \notin \bigcup_{j=0}^{r} D_{j}-\Omega_{1}
$$

Let $\tilde{\Omega}_{1}=\Omega_{1}$. Let

$$
k_{i}=\min \left\{k \in \mathbb{N} \cup\{0\}: F^{k}(x) \in \tilde{\Omega}_{i}\right\}
$$

for $1 \leq i \leq b$. Let $1 \leq i_{0} \leq b$ be such that $k_{i_{0}} \leq k_{i}$ for any $1 \leq i \leq b$. We claim that $F^{k_{i_{0}}}: \operatorname{Comp}_{x}\left(k_{i_{0}}, \tilde{\Omega}_{i_{0}}\right) \rightarrow \tilde{\Omega}_{i_{0}}$ is a conformal map, and hence we can take $\Omega=\tilde{\Omega}_{i_{0}}$ to complete the proof of the proposition.

We prove the claim by contradiction. Assume that such a map is not conformal. Let $A_{m}=\operatorname{Comp}_{F^{m}(x)}\left(k_{i_{0}}-m, \tilde{\Omega}_{i_{0}}\right)$ for $0 \leq m \leq k_{i_{0}}$. Then there will be a maximal $m_{0}<$ $k_{i_{0}}$ such that $A_{m_{0}}$ contains a critical point $e \in \omega(c)$. Then $F^{k_{i_{0}}-m_{0}}(e) \in \tilde{\Omega}_{i_{0}}$. So there is a positive integer $p$ such that $F^{k_{i_{0}}-m_{0}}(c)=B_{K}^{p}(c)$. Let $m_{0}<m_{1}<\cdots<m_{p}=k_{i_{0}}$ be the integers such that $B_{K}^{j}(c)=F^{m_{j}-m_{0}}(c)$ for any $0 \leq j \leq p$. Let $0 \leq i_{1} \leq b$ be such that $B_{I}^{p-1}(c)=F^{m_{p-1}-m_{0}}(c) \subset \tilde{\Omega}_{i_{1}}$. If $i_{1} \neq 1$, then $F^{m_{p-1}}(x) \in \tilde{\Omega}_{i_{1}}$, this contradicts the minimality of $k_{i_{0}}$. So $i_{1}=1$ and hence $F^{m_{p-1}}(x) \in \bigcup_{j=0}^{r} D_{j}$. Since there is no point in the forward orbit of $x$ which is contained in $\bigcup_{j=0}^{r} D_{j}-\Omega_{1}$, we have $F^{m_{p-1}}(x) \in \tilde{\Omega}_{1}=\Omega_{1}$, which contradicts the minimality of $k_{i_{0}}$ again.

Case 2. There is a non-negative integer $k$ such that

$$
F^{k}(x) \in \bigcup_{j=0}^{r} D_{j}-\Omega_{1}
$$

In this case we shall show that there is a univalent branch $h$ of $f^{-k^{\prime}}$ defined on $\Omega=\Omega_{1}$ or $\Omega_{2}$ such that $d(x, h(\Omega)) \leq C_{5} \operatorname{diam}(h(\Omega))$, where $k^{\prime}$ is the non-negative integer such that $F^{k}=f^{k^{\prime}}$ in a neighbourhood of $x$.

Let $P_{1}, P_{2}$ be admissible topological disks, we say that the triplet $\left(m, P_{1}, P_{2}\right)$ is bounded by a constant $C>1$ if $\left(P_{1}, P_{2}\right)$ is a $C$-bounded couple and if there is a piecewise smooth curve $\gamma$, joining $F^{m}(x)$ to $P_{1}$, such that

$$
l(\gamma) \leq C \operatorname{diam}\left(P_{1}\right)
$$

and such that for any $z \in \gamma$, we have

$$
B\left(z, C^{-1} \operatorname{diam}\left(P_{1}\right)\right) \cap \omega(c)=\emptyset .
$$

Remark 7.1. Let $\left(m, P_{1}, P_{2}\right)$ be a $C$-bounded triplet as before. Then $\left(m, P_{2}, P_{1}\right)$ is $C^{\prime}$-bounded for some $C^{\prime}>1$ depending only on $C$. Indeed, there is a curve $\rho$ joining $\partial P_{1}$ and $\partial P_{2}$, with $l(\rho) / \operatorname{diam}\left(P_{1}\right)$ uniformly bounded, such that for each point $z \in \rho$, $B\left(z, C^{-1} \operatorname{diam}\left(P_{1}\right)\right)$ is disjoint from $\omega(c)$. Let $\gamma^{\prime}=\gamma * \partial P_{1} * \rho$. This is a piecewise smooth curve joining $F^{m}(x)$ to $\partial P_{2}$, with similar properties as $\gamma$. 
Convention. For a Jordan curve we always give it an anti-clockwise orientation.

Lemma 7.6. Let $m$ be a non-negative integer and $P \subset V\left(F^{m}(x)\right)$ be a $C$-bounded admissible topological disk. Let $\rho \subset V\left(F^{m}(x)\right)-\omega(c)$ be a path with initial point $F^{m}(x)$ and endpoint $w \in \partial P$ with $l(\rho) \leq C \operatorname{diam}(P)$. Assume that for any $y \in \rho$, we have $B\left(y, C^{-1} \operatorname{diam}(P)\right) \cap \omega(c)=\emptyset$. Let $\rho^{\prime}$ be the lift of $\rho$ under $F^{m}$ with initial point $x$ and $z$ the endpoint of $\rho^{\prime}$. Assume that the lift of $\partial P$, considered as a loop based at $w$, with initial point $z$ is a closed Jordan curve. Then there is a constant $C^{\prime}$ depending only on $C$, and a topological disk $Q \subset U_{x}(m)$ such that $F^{m}: Q \rightarrow P$ is a well-defined conformal map and

$$
d(x, Q) \leq C^{\prime} \operatorname{diam}(Q) \text {. }
$$

Remark. There is a well-defined proper map $F^{m}: U_{x}(m) \rightarrow V\left(F^{m}(x)\right)$ and the lifts should be considered under the map.

Proof. Let $\gamma$ denote the lift of $\partial P$ with initial point $z$ and let $Q$ denote the topological disk bounded by $\gamma$, then $F^{m}: Q \rightarrow P$ is a conformal mapping. Let $h$ denote the inverse of this conformal mapping. The function $h$ has an analytic continuation along the path $\rho^{-1}$.

By the assumption, there is a uniformly bounded integer $p$, and round disks $B_{i}=$ $B\left(y_{i}, r_{i}\right), 1 \leq i \leq p$ which are disjoint from $\omega(c)$, such that

$$
\gamma \cup \partial P \subset \bigcup_{i=1}^{p} B\left(y_{i}, \frac{r_{i}}{2}\right) .
$$

From Koebe's principle, it follows that $h$ has a uniformly bounded distortion on $\gamma \cup \partial P$. Since $h$ is conformal on $P$, it also has uniformly bounded distortion on $P$. Thus the lemma holds.

Corollary 7.3. Let $P_{i}, i=1,2$ be admissible topological disks and let $m$ be a nonnegative integer. Assume that for $i=1,2$, there is a nice interval $A_{i} \subset P_{i}$ such that $P_{i} \cap \omega(c)=A_{i} \cap \omega(c)$. Assume also that the triplet $\left(m, P_{1}, P_{2}\right)$ is bounded by a constant $C>0$. If $\operatorname{diam}\left(P_{1}\right)$ is sufficiently small, then there is a constant $C^{\prime}$ depending only on $C$ and an integer $i \in\{1,2\}$ such that either of the following holds.

(1) There exists a topological disk $Q \subset U_{x}(m)$ such that $F^{m}: Q \rightarrow P_{i}$ is a conformal map and $d(x, h(Q)) \leq C^{\prime} \operatorname{diam}(Q)$.

(2) There is a positive integer $m^{\prime}<m$, two admissible topological disks $P_{i, 1}, P_{i, 2}$, such that the triplet $\left(m^{\prime}, P_{i, 1}, P_{i, 2}\right)$ is $C^{\prime}$-bounded. Moreover, there are nice intervals $A_{i, j} \subset P_{i, j}, j=1,2$ with the following properties:

(2.i) $A_{i, j} \supset P_{i, j} \cap \omega(c) \neq \emptyset, j=1,2$;

(2.ii) $f\left(A_{i, 1}\right)=f\left(A_{i, 2}\right), f\left(P_{i, 1}\right)=f\left(P_{i, 2}\right), F^{m-m^{\prime}}\left(P_{i, 1}\right)=P_{i}$;

(2.iii) $F^{m-m^{\prime}} \mid A_{i, 1}$ is monotone and $F^{m-m^{\prime}}\left(A_{i, 1}\right)=A_{i}$.

Moreover, $\operatorname{diam}\left(P_{i, 1}\right)$ is also very small.

Proof. The proof is a modification of McMullen's argument in his approach to the absence of a line field, see $[\mathbf{2 0}, \mathbf{2 1}]$. Let $\xi_{i}$ be a piecewise smooth curve from $F^{m}(x)$ to $\partial P_{i}$, 
$i=1,2$, such that for some $\eta>0$, some $C^{\prime}>1$, and each $y \in \xi_{i}$, we have

$$
B\left(y, \eta \operatorname{diam}\left(P_{1}\right)\right) \cap \omega(c)=\emptyset,
$$

and such that

$$
l\left(\xi_{i}\right) \leq C^{\prime} \operatorname{diam}\left(P_{1}\right) .
$$

By the definition of boundedness, such constants $\eta, C^{\prime}$ exist.

Let $\xi_{i}^{\prime}$ denote the lift of $\xi_{i}$ with initial point $x$ under $F^{m}, i=1,2$. Let $z_{i}$ denote the endpoint of $\xi_{i}^{\prime}$ and $\zeta_{i}$ denote the lift of $\partial P_{i}$ under $F^{m}$ with initial point $z_{i}$.

If either of $\zeta_{1}$ and $\zeta_{2}$ is a Jordan curve then we are in case 1 by Lemma 7.6. Assume we are not in this case. Let $m_{i}$ be the maximal integer such that $F^{m_{i}}\left(\zeta_{i}\right)$ is not a closed curve. Without loss of generality, assume that $m_{1} \geq m_{2}$. Let $\Delta_{i}$ denote the domain bounded by $F^{m_{i}+1}\left(\zeta_{i}\right)$, then both $\Delta_{1}$ and $\Delta_{2}$ intersect $\omega(c)$.

CLAim. The set $F^{m_{1}+1}\left(\zeta_{1} \cup \zeta_{2} \cup \xi_{1}^{\prime} \cup \xi_{2}^{\prime}\right)$ has a small diameter provided that diam $\left(P_{1}\right)$ is small.

Indeed, by construction, the map

$$
F^{m-m_{1}-1}: F^{m_{1}+1}\left(\zeta_{1} \cup \zeta_{2} \cup \xi_{1}^{\prime} \cup \xi_{2}^{\prime}\right) \rightarrow \partial P_{1} \cup \partial P_{2} \cup \xi_{1} \cup \xi_{2}
$$

is a conformal map. Since $\left(m, P_{1}, P_{2}\right)$ is bounded, the map has a uniformly bounded distortion. Thus, to prove this claim, it suffices to show the real trace of $\Delta_{1}$ is small when $\operatorname{diam}\left(P_{1}\right)$ is also small. But $\Delta_{1} \cap \mathbb{R}$ is an interval intersecting $\omega(c)$, and it is mapped diffeomorphically to a small interval $P_{1} \cap \mathbb{R}$, and so it must be small due to the absence of a wandering interval.

So we may assume that $m_{1}>m_{2}$. By the same reasoning, the set $F^{m_{1}}\left(\zeta_{1} \cup \zeta_{2} \cup \xi_{1}^{\prime} \cup \xi_{2}^{\prime}\right)$ is close to a critical point, say $e$, which is contained in the set $\omega(x)=\omega(c)$.

Let $U$ be a definite neighbourhood of $e$ such that $f \mid U: U \rightarrow f(U)$ is a branched covering with a unique critical point $e$ and let $\phi: U \rightarrow U$ be the prime transformation of the branched covering such that the lift of $\partial f(U)$, considered as a loop based at $f(z)(z \in \partial U)$, with initial point $z$ under $f$ is ended by $\phi(z)$.

Let

$$
\rho=\xi_{2} * \xi_{1}^{-1} * \partial P_{1} * \xi_{1} .
$$

Then $\rho$ is a piecewise smooth curve from $F^{m}(x)$ to $\partial P_{2}$. By choosing $\eta>0$ smaller, and $C^{\prime}$ larger, we may assume that

$$
l(\rho) \leq C^{\prime} \operatorname{diam}\left(P_{2}\right)
$$

and also that for any $y \in \rho$, we have

$$
B\left(y, \eta \operatorname{diam}\left(P_{2}\right)\right) \cap \omega(c)=\emptyset .
$$

Let $\rho^{\prime}$ be the lift of $\rho$ with initial point $x$ under $F^{m}$ and $z^{\prime}$ the endpoint of $\rho^{\prime}$.

Observation. The endpoint $F^{m_{1}}\left(z^{\prime}\right)$ of $F^{m_{1}}\left(\rho^{\prime}\right)$ is $\phi\left(F^{m_{1}}\left(z_{2}\right)\right)$. 
In fact, since the pull-back of $\xi_{i}$ with initial point $F^{m_{1}}(x)$ under $F^{m-m_{1}}$ is ended by $F^{m_{1}}\left(z_{i}\right)$, the pull-back of $\xi_{i}$ with initial point $\phi\left(F^{m_{1}}(x)\right)$ is ended by $\phi\left(F^{m_{1}}\left(z_{i}\right)\right)$, $i=1,2$. So the pull-back of $\xi_{1}^{-1}$ with initial point $\phi\left(F^{m_{1}}\left(z_{1}\right)\right)$ is $\phi\left(F^{m_{1}}(x)\right)$. The pullback of $\partial P_{1}$ with initial point $F^{m_{1}}(z)$ is ended by $\phi\left(F^{m_{1}}(z)\right)$ again by the definition of $\phi$. The observation follows.

Note also that the lift of $\partial P_{2}$ under $F^{m-m_{1}}$ with initial point $\phi\left(F^{m_{1}}\left(z_{2}\right)\right)$ is the Jordan curve $\phi\left(F^{m_{1}}\left(\zeta_{2}\right)\right)$, which bounds the topological disk $\phi\left(F^{m_{1}-m_{2}-1}\left(\Delta_{2}\right)\right)$. Let $P_{2,1}=$ $F^{m_{1}-m_{2}-1}\left(\Delta_{2}\right)$ and $P_{2,2}=\phi\left(P_{2,1}\right)$. Then $f\left(P_{2,1}\right)=f\left(P_{2,2}\right)$. If $P_{2,2} \cap \omega(c)=\emptyset$, then the lift of $\partial P_{2}$ under $F^{k}$ with initial point $z^{\prime}$ is obviously a Jordan curve and hence by Lemma 7.6, we are in case 1 . So let us assume $P_{2,2} \cap \omega(c) \neq \emptyset$. Obviously both $P_{2,1}$ and $P_{2,2}$ are admissible topological disks. Let $m^{\prime}=m_{1}$. Then it is easy to check that the triple ( $\left.m^{\prime}, P_{2,1}, P_{2,1}\right)$ is uniformly bounded using Koebe's Distortion Theorem. For $j=1,2$, let $A_{2, j} \subset P_{2, j}$ be the interval such that $F^{m-m_{1}}\left(A_{2, j}\right)=A_{2}$. It is easy to check properties (2.i)-(2.iii) for $i=2$.

We can complete the proof of Proposition 7.2 now.

Continuation of proof of Proposition 7.2. If $\Omega_{2} \cap \omega(c)=\varnothing$, then it follows from Lemma 7.6 that we can take $\Omega=\Omega_{2}$ to conclude the proof. So assume that $\Omega_{2} \cap \omega(c) \neq \emptyset$. Let $A_{i} \subset \Omega_{i}$ be the nice interval intersecting $\omega(c)$ such that $A_{i} \supset \Omega_{i} \cap \omega(c)$. The triplet $\left(k, \Omega_{1}, \Omega_{2}\right)$ is uniformly bounded. Recall that $A_{1}=K$ and $A_{2}$ is a monotone pull-back of $L$.

Applying Corollary 7.3 to the triplet $\left(k, \Omega_{1}, \Omega_{2}\right)$, we have two possibilities. If we are in case 1 in that corollary, then the proof is completed. Assume that we are in case 2. Then we have $i_{0} \in\{1,2\}$ and another triplet $\left(k_{1}, \Omega_{i_{0}}, \Omega_{i_{0} 2}\right)$ which is also uniformly bounded. Let $A_{i_{0} j}$ be the nice interval as in that corollary, $j=1,2$. Remember that both $A_{i_{0} 1}$ and $A_{i_{0} 2}$ are monotone pull-backs of $A_{i_{0}}$ and intersect $\omega(c)$.

Apply Corollary 7.3 to the triple $\left(k_{1}, \Omega_{i_{0}}, \Omega_{i_{0} 2}\right)$, and so on. Either we complete the proof within $N+1$ steps, or we will have $i_{0}, i_{1}, \ldots, i_{N} \in\{1,2\}$ and nice intervals

$$
A_{j}, A_{i_{0} j}, A_{i_{0} i_{1} j}, \ldots, A_{i_{0} i_{1} \cdots i_{N} j}, \quad(j=1,2),
$$

intersecting $\omega(c)$ such that for any $0 \leq s \leq N, A_{i_{0} i_{1} \cdots i_{s} 1}$ and $A_{i_{0} i_{1} \ldots i_{s} 2}$ are monotone pullbacks of $A_{i_{0} i_{1} \cdots i_{s}}$ which are symmetric with respect to a critical point in $\omega(c)$. For any $i \in\{1,2\}$, let $i^{\prime}$ denote the element of $\{1,2\}-\{i\}$. Let

$$
\mathbb{S}=\left\{A_{i_{0} i_{1}^{\prime}}, \ldots, A_{i_{0} i_{1} \cdots i_{N}^{\prime}}, A_{i_{0} i_{1} \cdots i_{N} 1}, A_{i_{0} i_{1} \cdots i_{N} 2}\right\} .
$$

Then $\mathbb{S}$ has $N+1$ elements, which are all monotone pull-backs of $A_{i_{0}}$. For each $S \in \mathbb{S}$, let $k(S)$ be the minimal positive integer such that $f^{k(S)}(c(S)) \in S$ for some $c(S) \in \omega(c)$ and let $A(S)$ be the pull-back of $S$ along the orbit $\left\{f^{j}(c(S))\right\}_{j=0}^{k(S)}$. Then $A(S)$ is a child of $A_{1}=K$ (if $i_{0}=1$ ) or $L$ (if $i_{0}=2$ ). It is easy to see that for $S, S^{\prime} \in \mathbb{S}$ with $S \neq S^{\prime}, A(S)$ and $A\left(S^{\prime}\right)$ are different. So we know that either $K$ or $L$ has at least $N+1$ children, which is a contradiction.

Acknowledgements. This paper was written during the author's postgraduate study at University of Tokyo and Hiroshima University. I am grateful to Professor Shishikura for 
his professional guidance and several valuable suggestions. I would also like to thank Professor van Strien for his interest in this work, and the referee for suggesting that I use less hyperbolic geometry. The author was supported by a Japanese Government Scholarship.

\section{REFERENCES}

[1] L. Ahlfors. Lectures on Quasi-conformal Maps. Van Nostrand, Princeton, NJ, 1966.

[2] B. Branner and J. H. Hubbard. The iteration of cubic polynomials I. Acta Math. 160 (1988), 143-206.

[3] B. Branner and J. H. Hubbard. The iteration of cubic polynomials II. Acta Math. 169 (1992), 229-325.

[4] P. Buser. Geometry and Spectra of Compact Riemann Surface. Birkhauser, Boston, 1992.

[5] L. Carelson and T. Gamelin. Complex Dynamics. Springer, Berlin, 1993.

[6] A. Douady and J. H. Hubbard. On the dynamics of polynomial-like mappings. Ann. Sci., Ec. Norm. Sup. $4^{e}$ Ser. 18 (1985), 287-343.

[7] J. Hu. Renormalization, rigidity, and universality in bifurcation theory. Thesis, 1995

[8] J. Hu and C. Tresser. Period doubling, entropy, and renormalization. Fund. Math. 155(3) (1998), $237-249$.

[9] J. H. Hubbard. Local connectivity of Julia sets and bifurcation loci: three theorems of J.-C. Yoccoz. Topological Methods in Modern Mathematics, A Symposium in Honor of John Milnor's 60th Birthday. Publish or Perish, Boston, MA, 1993.

[10] J. Graczyk and G. Swiatek. Generic hyperbolicity in the logistic family. Ann. Math. 146 (1997), 1-52.

[11] G. Levin and S. van Strien. Local connectivity of Julia sets of real polynomials. Ann. Math. 147 (1998), 471-541.

[12] G. Levin and S. van Strien. Total disconnectedness of the Julia set of real polynomials. Astérisque 261 (2000), xii, 161-172.

[13] M. Lyubich. On the Lebesgue measure of the Julia set of a quadratic polynomial. Stony Brook IMS Preprint 1991/10.

[14] M. Lyubich. Ergodic theory for smooth one dimensional dynamical systems. Stony Brook IMS Preprint 1991/11.

[15] M. Lyubich. Dynamics of quadratic polynomials, I and II. Acta Math. 178 (1997), 185-297.

[16] M. Lyubich. Renormalization Ideas in Conformal Dynamics (Current Developments in Mathematics, 1995). International Press, Cambridge, MA, 1994, pp. 155-190.

[17] M. Lyubich and J. Milnor. The unimodal Fibonacci maps. J. Amer. Math. Soc. 6 (1993), 425-457.

[18] R. Mañé, P. Sad and D. Sullivan. On the dynamics of rational map. Ann. Sci. Ec. Norm. Sup. 16 (1983), 193-217.

[19] M. Martens, W. de Melo and S. van Strien. The Julia-Fatou-Sullivan theory in one-dimensional dynamics. Acta Math. 168 (1992), 273-318.

[20] C. McMullen. Complex dynamics and renormalization. Annals of Mathematical Studies, Vol. 135, 1996.

[21] C. McMullen. Renormalization and 3-manifolds which fiber over the circle. Annals of Mathematical Studies, Vol. 142, 1994.

[22] C. McMullen and D. Sullivan. Quasiconformal homeomorphisms and dynamics III: The Teichmüller space of a holomorphic dynamical system. Adv. Math. 135 (1998), 351-395.

[23] J. Milnor. Dynamics in one complex variable: introductory lectures. Friedr. Vieweg and Sohn, Braunschweig, 1999

[24] J. Milnor. Local connectivity of Julia sets: expository lectures. The Mandelbrot Set, Theme and Variations (London Mathematical Society Lecture Notes, 274). Cambridge University Press, Cambridge, 2000, pp. 67-116.

[25] E. Prado. Ergodicity of conformal measures for unimodal polynomials. Conf. Geom. Dyn. 2 (1998), 29-44.

[26] W. Shen. Bounds for one-dimensional maps without inflection critical points. J. Math. Sci. Univ. Tokyo, to appear.

[27] M. Shishikura. Unpublished.

[28] M. Shishikura. Manuscript, 1998

[29] D. Sullivan. Bounds, quadratic differentials and renormalization conjectures. Amer. Math. Soc. Centennial Publications, Vol. 2, Mathematics into The Twenty-first Century (1988). American Mathematical Society, Providence, RI, 1988. 